\title{
JIANYI ZHOU
}

\section{Morphisme cellulaire et classes de Chern bivariantes}

Annales de la faculté des sciences de Toulouse $6^{e}$ série, tome $9, \mathrm{n}^{\mathrm{o}} 1$ (2000), p. 161-192

<http://www.numdam.org/item?id=AFST_2000_6_9_1_161_0>

(C) Université Paul Sabatier, 2000, tous droits réservés.

L'accès aux archives de la revue «Annales de la faculté des sciences de Toulouse » (http://picard.ups-tlse.fr/ annales/) implique l'accord avec les conditions générales d'utilisation (http://www.numdam.org/conditions). Toute utilisation commerciale ou impression systématique est constitutive d'une infraction pénale. Toute copie ou impression de ce fichier doit contenir la présente mention de copyright.

\section{NumDAM}

Article numérisé dans le cadre du programme Numérisation de documents anciens mathématiques http://www.numdam.org/ 


\title{
Morphisme cellulaire et classes de Chern bivariantes ${ }^{(*)}$
}

\author{
JIANYI ZHOU ${ }^{(1)}$
}

\begin{abstract}
RÉsumÉ. - Fulton et MacPherson ont conjecturé l'existence et l'unicité de classes de Chern en théorie bivariante. Brasselet et Sabbah ont donné chacun une démonstration de l'existence de ces classes. Nous montrons que leurs constructions coïncident, et donnons quelques résultats sur les morphismes cellulaires et sur l'obstruction d'Euler locale relative.
\end{abstract}

\begin{abstract}
Fulton and MacPherson conjectured the existence and uniqueness of Chern classes in bivariant theory. Brasselet and Sabbah have given two different constructions of such classes. We prove that these two constructions coincide and give some results on cellular morphisms and on relative Euler obstruction.
\end{abstract}

\section{Introduction}

La notion de théorie bivariante a été introduite par W. Fulton et R. MacPherson ( $[\mathrm{FM}])$. Une telle théorie fait correspondre à tout morphisme d'une catégorie convenable, un groupe abélien gradué muni de trois opérations : image réciproque, image directe et produit. On a comme exemples, l'homologie bivariante $H_{*}(X \stackrel{f}{\longrightarrow} Y)$, le groupe des fonctions constructibles

(*) Reçu le 26 avril 1999, accepté le 21 mars 2000

(1) Institut de Mathématiques de Luminy, Case 907, F-13288 Marseille Cedex 9.

e-mail: zhou@iml.univ-mrs.fr

ou Institute of Mathematics, Academia Sinica, 100080 Beijing (China).

e-mail: zhouyi@math08.math.ac.cn 
bivariantes $\mathcal{F}(X \stackrel{f}{\longrightarrow} Y)$, le groupe des cycles bivariants $\mathcal{Z}(X \stackrel{f}{\longrightarrow} Y)$ etc. Lorsqu'on spécialise cette théorie aux morphismes $X \stackrel{i d}{\longrightarrow} X$ et $X \longrightarrow p t$ on obtient les théories "classiques", par exemple $H_{*}(X \stackrel{i d}{\longrightarrow} X)$ est la cohomologie de $X$ et $H_{*}(X \longrightarrow p t)$ est son homologie.

Une transformation d'une théorie bivariante dans une autre respectant les trois opérations bivariantes est appelée transformation de Grothendieck. W. Fulton et R. MacPherson ont ainsi montré l'existence et l'unicité des classes de Stiefel-Whitney bivariantes, c'est-à-dire, d'une transformation de Grothendieck $\mathcal{F} \longrightarrow H$ à coefficients $\mathbf{Z}_{2}[\mathrm{FM}, \S 6]$. Ils ont conjecturé l'existence et l'unicité des classes de Chern bivariantes. Si la réponse est positive, en utilisant les propriétés de la théorie bivariante, on obtient systématiquement des formules de type Riemann-Roch, par exemple des formules de type Verdier-Riemann-Roch pour la transformation de Verdier-Radon ([EOY, Yo1, Yo2]), et des théories de spécialisation des classes de StiefelWhitney et de Chern [Ve1].

J.-P. Brasselet a construit une théorie de classes de Chern bivariantes [Br1] par des méthodes topologiques inspirées de celles de M.H. Schwartz et dans la catégorie des espaces analytiques réduits admettant une décomposition cellulaire.

La construction de C. Sabbah [Sab3] très différente suppose outre la propreté des morphismes considérés, l'existence d'un plongement relatif de $X$ dans une variété analytique, ainsi que des conditions techniques "d'orientabilité relative" [Sab3, §4.5] (cf. $\S 2.7$ et $\S 3.3$ ). Cette dernière hypothèse est toujours réalisée si la base $S$ est une courbe lisse. C. Sabbah a construit une transformation de Grothendieck $E u_{f}$ du groupe des cycles bivariants $\mathcal{Z}(X \stackrel{f}{\longrightarrow} S)$ vers le groupe des fonctions constructibles bivariantes $\mathcal{F}(X \stackrel{f}{\longrightarrow} S)$, dite "obstruction d'Euler locale relative". Cette transformation donne pour chaque morphisme $f$ un isomorphisme de groupes abéliens $E u_{f}: \mathcal{Z}(X \stackrel{f}{\longrightarrow} S) \longrightarrow \mathcal{F}(X \stackrel{f}{\longrightarrow} S)[$ Sab3, §3]. Si le morphisme $f: X \longrightarrow S$ est propre et $S$ est une courbe analytique lisse, à toute fonction constructible bivariante $\alpha$, on peut associer une classe $c_{B}(\alpha)$ à la Brasselet d'une part et une classe $c_{S}(\alpha)$ à la Sabbah d'autre part. Il est donc naturel de comparer $c_{B}(\alpha)$ (composé avec $E u_{f}$ ) avec $c_{S}(\alpha)$. Le problème de l'unicité reste toujours entier.

Dans tout ce travail, un espace analytique signifie complexe réduit et un morphisme analytique sera un morphisme analytique complexe entre espaces analytiques complexes réduits. On suppose que tous les morphismes sont propres. Fixons les notations : $f: X \longrightarrow S$ sera un morphisme analytique 
propre avec $S$ une courbe lisse, et $X$ sera plongé relativement dans une variété analytique $M$, c'est-à-dire, dans le produit $S \times M$. Notons $G_{d}(T M)$ l'espace fibré en grassmaniennes des $d$-plans tangents à $M$. Dans ce qui suit nous prouvons le résultat suivant :

THÉORÈME PRINCIPAL. - Pour toute fonction constructible bivariante $\alpha$ du morphisme considéré $f: X \longrightarrow S$, notons $Z=E u_{f}^{-1}(\alpha)$ le cycle bivariant associé. On suppose que, pour un entier $d$ convenable, le morphisme naturel $S \times G_{d}(T M) \longrightarrow S \times M$ admet une décomposition cellulaire compatible avec le plongement $N_{f}(Z) \longleftrightarrow S \times G_{d}(T M)$ du transformé de Nash relatif. Alors les classes de Chern bivariantes de Brasselet et de Sabbah coïncident

$$
c_{B}(\alpha)=c_{S}(\alpha)
$$

Dans le premier paragraphe, nous rappelons des notions utiles. Dans le second paragraphe, nous décrivons d'abord les propriétés des morphismes cellulaires. Nous construisons un homomorphisme d'Alexander en théorie bivariante, qui induit par restriction aux fibres, l'isomorphisme d'Alexander usuel. Nous rappelons ensuite la construction de J.-P. Brasselet. La classe fondamentale bivariante topologique est définie à la fin de ce paragraphe. Le paragraphe 3 est consacré à la construction de C. Sabbah. En se donnant un théorème de proportionnalité entre l'obstruction d'un champ de repères relatifs et celle de son relevé dans le transformé de Nash relatif $(\S 4.1)$, une construction topologique de classe de Chern bivariante d'un fibré (\$4.2), nous montrons dans le paragraphe 4 la coïncidence des classes bivariantes de Brasselet et de Sabbah.

Je tiens à remercier J.-P. Brasselet d'avoir dirigé ce sujet pendant la préparation de ma thèse. Je tiens à remercier M. Merle, C. Sabbah, J. Tapia et M. Kwieciński pour leurs nombreuses discutions et remarques sur ce sujet.

\section{Rappels et notations}

\subsection{Homologie bivariante}

Dans ce paragraphe, on rappelle la notion d'homologie bivariante, définie dans [FM, §3].

1.1.1. Considérons la catégorie des espaces topologiques $X$ qui admettent un plongement fermé dans une variété paracompacte de classe $C^{1}$ et orientable. 
Remarquons que les espaces considérés sont localement compacts. On dispose donc dans ce cadre du formalisme de dualité de Verdier [Ve3] pour les faisceaux de Z-modules. Soit $f: X \longrightarrow Y$ un morphisme, suivant Fulton et MacPherson [FM, $\S 7.3$ ] le groupe

$$
H^{i}(X \stackrel{f}{\longrightarrow} Y)=R^{i} H o m\left(R f_{!} \mathbf{Z}_{X}, \mathbf{Z}_{Y}\right)=H^{i}\left(X, f^{!} \mathbf{Z}_{Y}\right) .
$$

est appelé homologie bivariante de $f: X \longrightarrow Y$.

Soit de plus $\phi: X \longrightarrow M$ un plongement fermé où $M$ est une variété de classe $C^{1}$ et orientable de dimension $n$, alors le morphisme $(f, \phi): X \longrightarrow$ $Y \times M$ est un plongement fermé. Nous noterons $X_{\phi}$ l'image de $X$ par ce dernier. On dispose alors d'un isomorphisme naturel [FM, §3]

$$
H^{i}(X \stackrel{f}{\longrightarrow} Y) \cong H^{i+n}\left(Y \times M,(Y \times M) \backslash X_{\phi}\right) .
$$

En particulier, $H^{i}(X \stackrel{i d}{\longrightarrow} X)$ s'identifie canoniquement à $H^{i}(X)$ et $H^{-i}(X \longrightarrow p t)$ à $H_{i}(X)$, où $H^{*}(X)$ et $H_{*}(X)$ désignent la cohomologie et l'homologie singulières à coefficients dans $\mathbf{Z}[\mathrm{FM}, \S 3]$.

On a les trois opérations bivariantes rappelées ci-dessous, elles sont compatibles entre elles dans les cas où cela a un sens.

1.1.2. Image réciproque. La formation des $H^{i}(X \stackrel{f}{\longrightarrow} Y)$ est fonctorielle au sens suivant :

Si le diagramme

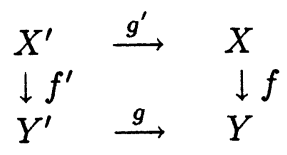

est un produit fibré, on a un morphisme image réciproque

$$
g^{*}: H^{i}(X \stackrel{f}{\longrightarrow} Y) \longrightarrow H^{i}\left(X^{\prime} \stackrel{f^{\prime}}{\longrightarrow} Y^{\prime}\right)
$$

avec les propriétés évidentes de fonctorialité.

1.1.3. Image directe. Si on a un composé de deux morphismes $X^{\prime} \stackrel{g}{\longrightarrow} X \stackrel{f}{\longrightarrow} Y$, avec $g$ un morphisme propre, on a un morphisme image directe

$$
g_{*}: H^{i}\left(X^{\prime} \stackrel{f \circ g}{\longrightarrow} Y\right) \longrightarrow H^{i}(X \stackrel{f}{\longrightarrow} Y),
$$

avec ici aussi les propriétés évidentes de fonctorialité. 
1.1.4. Produit bivariant. Lorsque l'on a un composé de deux morphismes $X \stackrel{f}{\longrightarrow} Y \stackrel{g}{\longrightarrow} Z$, on a un morphisme de produit bivariant associatif :

$$
H^{i}(X \stackrel{f}{\longrightarrow} Y) \otimes H^{j}(Y \stackrel{g}{\longrightarrow} Z) \longrightarrow H^{i+j}(X \stackrel{g \circ f}{\longrightarrow} Z) .
$$

\subsection{Fonctions constructibles bivariantes}

1.2.1. On considère $f: X \longrightarrow Y$ un morphisme analytique. Notons $\mathcal{F}(X)$ le groupe des fonctions constructibles analytiques sur $X$. Le groupe $\mathcal{F}(X \longrightarrow Y)$ des fonctions constructibles bivariantes associé à $f$ est le sousgroupe de $\mathcal{F}(X)$ des fonctions satisfaisant à la condition d'Euler locale suivante :

Soit $\alpha$ une fonction constructible. Etant donné un sous-ensemble $A$ de $X$, la caractéristique d'Euler pondérée par $\alpha$ est $\chi(A, \alpha):=\sum_{i \in \mathbf{Z}} \chi\left(A \cap \alpha^{-1}(i)\right)$. Pour tout $x_{0} \in X$, on choisit un plongement local $\left(X, x_{0}\right) \longrightarrow\left(\mathbf{C}^{n}, 0\right)$. Si $B_{\varepsilon}$ est la boule de $\mathbf{C}^{n}$ centrée en 0 et de rayon $\varepsilon$, alors pour $\varepsilon>0$ assez petit, et pour tout $y \in Y$ assez proche de $f\left(x_{0}\right)$, la caractéristique d'Euler pondérée par $\alpha: \chi\left(B_{\varepsilon} \cap f^{-1}(y), \alpha\right)$ ne dépend pas du point $y$ choisi et vaut $\alpha\left(x_{0}\right)$.

Pour les définitions précises des opérations bivariantes, voir $[\mathrm{FM}, \S 6]$ dans le cas des coefficients $\mathbf{Z}_{2}$ et $[\mathrm{Br} 1, \S 2]$ ou $[\mathrm{Sab3}, \S 1]$ dans le cas des coefficients entiers.

1.2.2. Rappelons qu'une transformation de Grothendieck de $\mathcal{F}$ dans $H$ est une collection d'homomorphismes de groupes abéliens $\mathcal{F}(X \longrightarrow Y) \longrightarrow$ $H(X \longrightarrow Y)$ compatible avec les trois opérations des théories bivariantes. W. Fulton et R. MacPherson ont énoncé la conjecture suivante :

CONJECTURE ([FM,§10.4]). - Il existe une unique transformation de Grothendieck $c$ de $\mathcal{F}$ dans $H$ telle que, pour le morphisme $X \longrightarrow p t$ où $X$ est une variété lisse, $c\left(1_{X}\right)=c(T X) \cap[X]$, où $1_{X} \in \mathcal{F}(X \longrightarrow p t)$ associe à tout point de $X$ la valeur $1 ; c(T X)$ désigne la classe de Chern du fibré tangent à $X$ et $[X]$ est la classe fondamentale de $X$.

L'image d'une fonction constructible $\alpha$ par une telle transformation est appelée classe de Chern bivariante de $\alpha$. 


\subsection{Espace conormal relatif et modification de Nash relative}

Rappelons ici les notions d'espace conormal relatif et de transformé de Nash relatif.

1.3.1. Soit $f: X \longrightarrow Y$ un morphisme analytique de dimension relative $d$, l'espace analytique $X$ étant plongé dans une variété analytique $M$ de dimension $m$. Soit $t$ un entier compris entre $d$ et $m-1$, on notera $G_{t}(T M)$ la variété fibrée en grassmanniennes des $t$-plans de l'espace tangent $T M$. Un point de $G_{t}(T M)$ est identifié à un couple $(x, H)$ avec $x$ un point de $M$ et $H$ un $t$-plan tangent à $M$ en $x$. Si $X^{0}$ désigne l'ouvert dense de $X$ sur lequel $f$ est lisse, on notera $G_{t}\left(\left.f\right|_{X^{0}}\right)$ le sous-espace analytique de $G_{t}(T M)$ constitué des couples $(x, H)$ tels que $x \in X^{0}$, et que $H$ contient l'espace tangent relatif $T_{x}\left(\left.f\right|_{X^{0}}\right)=T_{x}\left(f^{-1}(f(x))\right)$. On note $G_{t}\left(\left.f\right|_{X}\right)$ l'adhérence de $G_{t}\left(\left.f\right|_{X^{0}}\right)$ dans $G_{t}(T M)$. C'est un espace analytique. De plus, le morphisme de projection $\pi: G_{t}\left(\left.f\right|_{X^{0}}\right) \longrightarrow X^{0}$ se prolonge de manière unique en un morphisme propre noté encore $\pi: G_{t}\left(\left.f\right|_{X}\right) \longrightarrow X$. L'espace analytique $G_{t}\left(\left.f\right|_{X}\right)$ est muni d'un fibré $E^{t}$ de rang $t$ dont la fibre en un point $(x, H)$ est le $t$-plan $H$.

1.3.2. Le fibré projectif $\mathbf{P} T^{*} M$ associé au fibré cotangent s'identifie naturellement au fibré en grassmanniennes des hyperplans tangents à $M$. On note $C\left(\left.f\right|_{X}, M\right)$ l'espace analytique $G_{m-1}\left(\left.f\right|_{X}\right)$ et on l'appelle espace conormal relatif, il est muni d'une projection notée $\tau_{f}: C\left(\left.f\right|_{X}, M\right) \longrightarrow X$.

1.3.3. Remarquons que les fibres de $\tau_{f}$ sont de dimension au moins $m-d-1$. Notons avec ([HMS], [Sab1]), pour chaque entier $k$ compris entre 0 et $d$,

$$
\Sigma_{k}(f)=\left\{x \in X \mid \operatorname{dim}\left(\tau_{f}^{-1}(x)\right) \geqslant m-d+k-1\right\} .
$$

C'est un sous-espace analytique qui est défini comme le lieu où la $k$-ème variété polaire locale relative est non vide (voir [LT, Te2] pour la notion de variété polaire).

DÉFINITION ([HMS], [Sab1]). - Le morphisme $f$ est dit sans éclatement en codimension 0 , si les fibres de $\left.f\right|_{\Sigma_{k}(f)}: \Sigma_{k}(f) \longrightarrow Y$ sont de dimension au plus $d-k$.

1.3.4. Dans $\S 1.3 .1$, lorsqu'on pose $t=d$, on a le transformé de Nash relatif : $N_{f}(X)=G_{d}\left(\left.f\right|_{X}\right)$ avec la projection $\nu_{f}: N_{f}(X) \longrightarrow X$. C'est une modification propre de $X$. Sur $N_{f}(X)$, on a le fibré de Nash relatif $\theta$ de rang $d$. 


\subsection{Stratification de Thom}

1.4.1. Soit $f: X \longrightarrow Y$ un morphisme analytique. Supposons que le morphisme $f$ soit stratifié, i.e. qu'il y ait une stratification $\left(X_{i}\right)$ de $X$ et une stratification $\left(Y_{j}\right)$ de $Y$ telles que pour chaque strate $X_{i}$, la restriction $\left.f\right|_{X_{i}}$ soit une submersion surjective sur une strate de $Y$. Supposons que $X$ soit irréductible, et notons $X^{0}$ la strate dense de $X$. L'espace tangent en $x$ à la fibre $X_{i, f(x)}=f^{-1}(f(x)) \cap X_{i}$, noté $T_{x}\left(\left.f\right|_{X_{i}}\right)$ est appelé espace tangent relatif.

Considérons une strate $X_{i}$, elle est contenue dans l'adhérence de $X^{0}$.

1.4.2. Définition (Thom). - On dira que le couple $\left(X^{0}, X_{i}\right)$ satisfait la condition $A_{f}$ au point $x$ de $X_{i}$ si pour toute suite $\left(x_{n}\right)$ de $X^{0}$ tendant vers $x$, on a, quand la limite existe,

$$
\lim _{n \rightarrow+\infty} T_{x_{n}}\left(\left.f\right|_{X^{0}}\right) \supset T_{x}\left(\left.f\right|_{X_{i}}\right)
$$

On dira que le couple $\left(X^{0}, X_{i}\right)$ satisfait la condition $A_{f}$, si cette condition est satisfaite en tout point de $X_{i}$.

1.4.3. Définition (voir $[\mathrm{Sab} 1, \S 1.3]$ ). - On dira que le morphisme $f$ admet une bonne stratification s'il existe une stratification analytique complexe $\left(X_{i}\right)$ de $X \backslash X^{0}$, telle que pour tout $X_{i}$, le couple $\left(X^{0}, X_{i}\right)$ vérifie la propriété $A_{f}$ de Thom.

1.4.4. H. Hironaka ([Hi2, Th.1, §5], voir aussi [HMS, Th.4.1.3]) a donné une condition suffisante pour l'existence d'une bonne stratification. C. Sabbah ([Sab1, Th.1.3.1]) a prouvé que la condition de non-éclatement en codimension 0 de $f$ est nécessaire et suffisante pour avoir une bonne stratification.

Dans [HMS,§4], est précisée la conséquence suivante du théorème d'Hironaka qui décrit le comportement d'espaces conormaux par changement de base. Pour ce fait, soit $\pi: \hat{Y} \longrightarrow Y$ un morphisme analytique. Notons $\hat{C}=C\left(\left.f\right|_{X}, M\right) \times_{Y} \hat{Y}$ et $\hat{X}=X \times_{Y} \hat{Y}$ les produits fibrés, $\hat{\tau}: \hat{C} \longrightarrow \hat{X}$ et $\hat{f}: \hat{X} \longrightarrow \hat{Y}$ les morphismes naturels.

LEMME ([HMS,§4]). - Si le morphisme $f$ est sans éclatement en codimension 0 , alors chaque composante irréductible $\Gamma$ de $\hat{C}$ s'identifie avec l'espace conormal relatif au morphisme $\hat{f}: \hat{\tau}(\Gamma) \longrightarrow \hat{Y}$ (pour le plongement naturel de $\hat{X}$ dans $\left.M \times_{Y} \hat{Y}\right)$.

1.4.5. Remarque ([Sab1]). - Si $f: X \longrightarrow S$ est un morphisme propre analytique et $S$ est une courbe lisse, alors $f$ admet une bonne stratification. 


\section{Construction de Brasselet, relèvement de champ de repères relatifs}

\subsection{Décomposition cellulaire}

2.1.1. On désignera par $e^{n}$ la boule fermée euclidienne unitaire de $\mathbf{R}^{n}$ et par $\partial e^{n}$ la sphère euclidienne unitaire de $\mathbf{R}^{n}$. Une décomposition cellulaire $K$ d'un espace topologique $X$ est la donnée

a) d'un recouvrement fermé et localement fini $K$ de $X$ dont les éléments sont appelés cellules;

b) pour chaque $\sigma \in K$ d'un homéomorphisme $\phi: e^{n} \longrightarrow \sigma$ pour un $n$ convenable appelé dimension de $\sigma$, tel que $\phi\left(\partial e^{n}\right)$ soit le bord de $\sigma$ (en tant que variété à bord), noté $\partial \sigma$. L'intérieur d'une cellule est appelé cellule ouverte. Les cellules ouvertes forment une partition de $X$.

c) Si $\sigma, \sigma^{\prime} \in K$, l'intersection $\sigma \cap \sigma^{\prime}$ est une cellule si $\sigma \cap \sigma^{\prime} \neq \emptyset$.

d) Si $\sigma \in K$, le bord $\partial \sigma$ est une réunion finie de cellules.

Soient $\sigma$ et $\sigma^{\prime}$ deux cellules, on dit que $\sigma^{\prime}$ est une face de $\sigma$, et on note $\sigma^{\prime} \prec \sigma$, si $\sigma^{\prime}=\sigma$ ou $\sigma^{\prime} \subset \partial \sigma$. Remarquons que si $\sigma^{\prime}$ est une face de $\sigma$ alors $\operatorname{dim}\left(\sigma^{\prime}\right) \leqslant \operatorname{dim}(\sigma)$.

On obtient ainsi un CW-complexe de type particulier. Les axiomes b) et c) n'étant en général pas exigés dans la définition d'un CW-complexe.

2.1.2. Dans toute la suite, on exigera, lorsque $X=M$ est une variété de classe $C^{1}$, que les homéomorphismes $\phi$ sont de classe $C^{1}$.

De plus, on supposera avoir choisi un point $\hat{\sigma}$ à l'intérieur $\stackrel{\circ}{\sigma}$ de chaque cellule $\sigma$. Le point $\hat{\sigma}$ est appelé centre de $\sigma$ (si la dimension de $\sigma$ est nulle, $\sigma$ est un point, $\stackrel{\circ}{\sigma}=\sigma$ et $\hat{\sigma}=\sigma$ ). Etant donnée une cellule $\sigma$ de $K$, à toute suite $\sigma_{1} \prec \sigma_{2} \prec \cdots \prec \sigma_{k}$ de faces incidentes strictes de $\sigma$, on associe un simplexe dans $\sigma$ de sommets $\hat{\sigma}_{1}, \hat{\sigma}_{2}, \cdots, \hat{\sigma}_{k}$. On notera $\left\langle\hat{\sigma}_{1}, \hat{\sigma}_{2}, \cdots, \hat{\sigma}_{k}\right\rangle$ le simplexe associé. On obtient ainsi une subdivision $\Delta(K)$ appelée subdivision barycentrique de $K$, ce qui permet de construire, classiquement, une décomposition en cellules duales $D(K)$ de $K$. Pour toute cellule $\sigma$ de $K$, la cellule duale $D(\sigma)$ est la réunion

$$
D(\sigma):=\bigcup\{\delta \in \triangle(K) \mid \delta \cap \sigma=\hat{\sigma}\} .
$$

On a $D(\sigma) \cap \sigma=\{\hat{\sigma}\}$. 
Morphisme cellulaire et classes de Chern bivariantes

2.1.3. Décomposition cellulaire d'un espace stratifié.

Soit $\left(X_{i}\right)$ une stratification d'un espace analytique $X$ et $K$ une décomposition cellulaire de $X$. On dit que la décomposition cellulaire $K$ est compatible avec la stratification $\left(X_{i}\right)$ si toute cellule ouverte de $K$ est contenue dans une seule strate.

\subsection{Morphisme cellulaire}

2.2.1. Soit $f: X \longrightarrow Y$ un morphisme analytique et $K$ (resp. $L$ ) une décomposition cellulaire de $X$ (resp. $Y$ ). On dit que $f$ est cellulaire si pour toute cellule $\sigma$ de $K$,

a) $\tau=f(\sigma) \in L$;

b) $f(\stackrel{\circ}{\sigma}) \subset \stackrel{\circ}{\tau}$ et $\left.f\right|_{\stackrel{\circ}{\sigma}}: \stackrel{\circ}{\sigma} \longrightarrow \stackrel{\circ}{\tau}$ est une submersion.

Notons $\operatorname{dim}_{f}(\sigma)=\operatorname{dim}(\sigma)-\operatorname{dim}(f(\sigma))$ la dimension relative de la cellule $\sigma$.

2.2.2. Remarque. - On a les propriétés suivantes,

1) D'après le premier lemme d'isotopie de Thom, $f$ induit une fibration triviale de $\stackrel{\sigma}{\circ}$ sur $\stackrel{\circ}{\tau}$.

2) $\operatorname{Sidim}(\sigma)=0$, alors $f(\partial \sigma)=\partial(f(\sigma))$, dans ce cas, on dira que $\sigma$ est $f$-horizontale.

Si $\operatorname{dim}_{f}(\sigma)>0$, alors $f(\partial \sigma)=f(\sigma)$ et $\sigma$ admet des faces $f$-horizontales sur $f(\sigma)$.

3) Comme conséquence de 1), la trace de $K$ sur chaque fibre $X_{y}$ de $f$ définit une décomposition cellulaire de $X_{y}$.

4) Quitte à raffiner $K$, on peut supposer que pour chaque face $\tau^{\prime}$ de $\tau=f(\sigma)$, la trace $\sigma^{\prime}=\overline{\sigma \cap f^{-1}\left(\dot{\tau}^{\prime}\right)}$ est une face de $\sigma$. Par abus de notation, $\sigma \cap f^{-1}\left(\tau^{\prime}\right)$ signifiera la cellule $\sigma^{\prime}$, dite face dominante de $\sigma$ sur $\tau^{\prime}$ (voir Fig. 1).

2.2.3. Supposons de plus que le morphisme $f$ soit stratifié. On dira que le morphisme stratifié $f$ est cellulaire si :

a) $K$ (resp. $L)$ est compatible avec les strates de $X($ resp. $Y)$;

b) $f$ est cellulaire. 

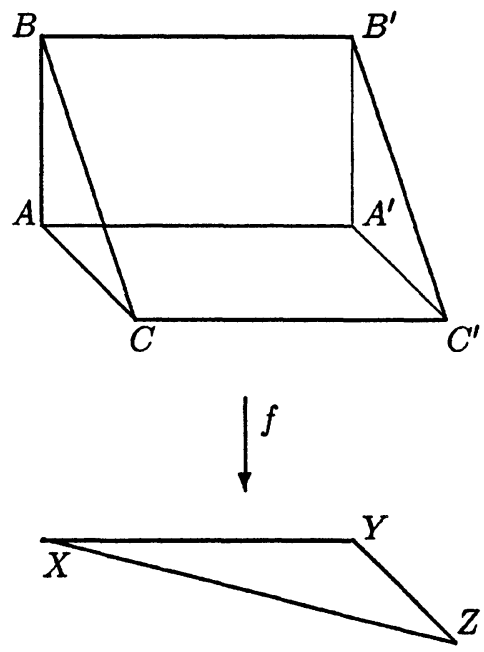

Fig. 1.

$\tau=X Y Z, \sigma=A B C A^{\prime} B^{\prime} C^{\prime} . \tau^{\prime}=X Y, \sigma^{\prime}=A A^{\prime} B B^{\prime}$ et $\sigma_{1}=A B C$.

L'application $f$ est telle que $f\left(\sigma_{1}\right)=\{X\}, f\left(A^{\prime} B^{\prime} C^{\prime}\right)=Y Z$ et que $f\left(\sigma^{\prime}\right)=\tau^{\prime}$. On a $f^{-1}\left(\tau^{\prime}\right)=\sigma^{\prime} \cup \sigma_{1}$. Mais, la face dominante sur $\tau^{\prime}$ est $\sigma^{\prime}=\overline{f^{-1}\left(\tau^{\prime}\right) \cap \sigma}$.

2.2.4. Considérons un morphisme $f: X \longrightarrow Y$ stratifié et cellulaire. On suppose de plus que l'on s'est donné un plongement fermé $\phi: X \longrightarrow M$, où $M$ est lisse et munie d'une décomposition cellulaire que l'on notera encore $K$ induisant la décomposition cellulaire $K$ de $X$. On suppose avoir choisi pour chaque cellule de $K$ (resp. $L$ ) un centre (voir §2.1.2) de sorte que $f$ envoie centre sur centre. On obtient ainsi des subdivisions barycentriques $\triangle(K)$ et $\triangle(L)$ de telle sorte que, pour tout simplexe de $\Delta(L)$, son image inverse par $f$ soit une réunion des simplexes de $\Delta(K)$ (voir Fig. 2).

Ces conditions impliquent que les simplexes de $\Delta(K)$ sont les plus "verticaux" possibles. Par exemple, si les cellules $\sigma_{1} \prec \sigma_{2} \prec \cdots \prec \sigma_{p}$ ont tous pour image $\tau$, alors le simplexe $\left\langle\hat{\sigma}_{1}, \hat{\sigma}_{2}, \cdots, \hat{\sigma}_{p}>\right.$ s'envoie sur le centre $\hat{\tau}$.

2.2.5. Lemme. - Le morphisme analytique $f: X \longrightarrow S$ où $S$ est une courbe lisse, possède une décomposition cellulaire.

Démonstration. - D'après le théorème de Teissier [Te1], pour tout morphisme $f$, il existe une modification $\pi: \widetilde{S} \longrightarrow S$ composée d'une suite 
d'éclatements, telle que le morphisme $\tilde{f}: \tilde{X} \longrightarrow \widetilde{S}$ obtenu par le changement de base par $\pi$ soit triangulable. Or toute modification de ce type d'une courbe lisse est un isomorphisme. On en déduit que $f$ lui-même est triangulable et donc possède une décomposition cellulaire.
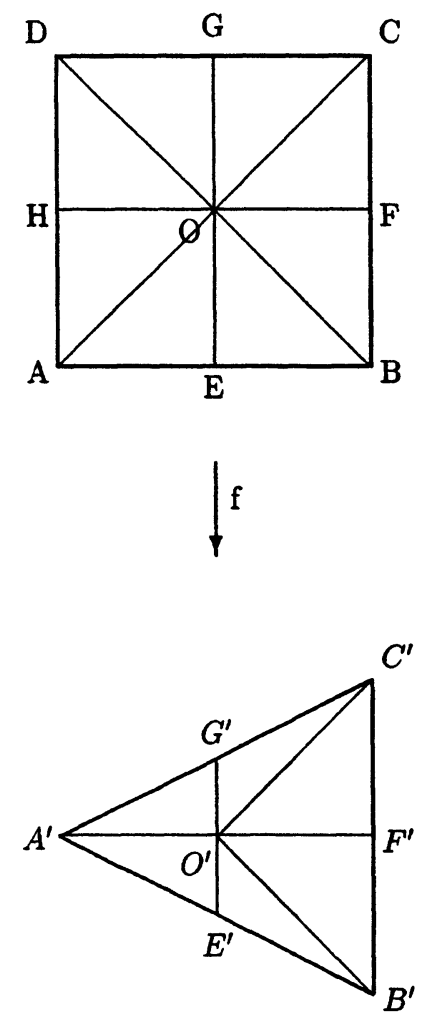

Fig. 2.

Dans cet exemple, notons $\tau=A^{\prime} B^{\prime} C^{\prime}$ et $\sigma=A B C D$.

L'application $f$ est telle que $f(A D)=\left\{A^{\prime}\right\}$. Le simplexe $H O$ a $A^{\prime} O^{\prime}$ pour image et l'image du simplexe $D O$ est contenue dans $A^{\prime} G^{\prime} O^{\prime}$.

2.3. Par la suite, on décrit la construction de cellule duale relative [ $\mathrm{Br} 1, \S 4]$.

\subsubsection{Centre relatif $C_{f}(\sigma)$.}

Soit $\sigma$ une cellule de $K$ contenue dans $X$, on construit d'abord le centre relatif $C_{f}(\sigma)$ tel que pour chaque point $y$ situé à l'intérieur de $\tau=$ 


\section{Jianyi Zhou}

$f(\sigma)$, l'intersection $C_{f}(\sigma) \cap \sigma_{y}$ soit un point $\hat{\sigma}_{y}$ appelé centre de $\sigma_{y}$. Par conséquent, l'intérieur de $C_{f}(\sigma)$ est homéomorphe à $\stackrel{\circ}{\tau}$. Il est un relèvement de $\tau$ dans $\sigma$.

Pour cela, considérons toutes les séquences possibles de faces de $\tau$ :

$$
\tau_{0} \prec \tau_{1} \prec \cdots \prec \tau_{p}
$$

avec $\operatorname{dim}\left(\tau_{i}\right)<\operatorname{dim}\left(\tau_{i+1}\right)$ pour $0 \leqslant i<p$ et la cellule $s_{i}$ dans $f^{-1}\left(\tau_{i}\right) \cap \sigma$ est de dimension la plus grande possible telle que :

$$
s_{0} \prec s_{1} \prec \cdots \prec s_{p} .
$$

On considère alors, toutes les séquences possibles de faces de $\sigma$ :

$$
\sigma_{0} \prec \sigma_{1} \prec \cdots \prec \sigma_{q}
$$

pour lesquelles $\operatorname{dim}\left(\sigma_{k}\right)<\operatorname{dim}\left(\sigma_{k+1}\right)$, et les $\sigma_{k}$ sont des $s_{i}$. Le centre relatif $C_{f}(\sigma)$ est la réunion de tous les simplexes associés $\left\langle\hat{\sigma}_{0}, \hat{\sigma}_{1}, \cdots, \hat{\sigma}_{q}\right\rangle$.

2.3.2. La cellule duale relative $A_{f}(\sigma)$ est définie comme la réunion $A_{f}(\sigma):=\bigcup\left\{\delta \in \triangle(K) \mid \delta \cap \sigma \subset C_{f}(\sigma)\right.$, et si $\delta \subset \sigma_{i}$, alors $\left.\sigma \prec \sigma_{i}\right\}$.

On note $B_{f}(\sigma)$ le bord de $A_{f}(\sigma)$ et $A_{f}^{\prime}(\sigma)$ (resp. $B_{f}^{\prime}(\sigma)$ ) la trace de $A_{f}(\sigma)$ (resp. $B_{f}(\sigma)$ ) dans $f^{-1}(\stackrel{\circ}{\tau})$ avec $\tau=f(\sigma)$. On remarque que $B_{f}^{\prime}(\sigma)$ est le bord "relatif" de $A_{f}^{\prime}(\sigma)$, c'est-à-dire, la réunion de bords des fibres $A_{f}^{\prime}(\sigma)_{y}=A_{f}^{\prime}(\sigma) \cap f^{-1}(y)$ pour $y \in \stackrel{\circ}{\tau}$.

2.3.3. Lemme. ([Br1, §4. Lemme 3])

1) $A_{f}^{\prime}(\sigma), B_{f}^{\prime}(\sigma)$ sont des produits sur $\stackrel{\tau}{\tau}$.

2) $B_{f}(\sigma)=\bigcup_{i} A_{f}\left(\sigma_{i}\right)$ où $i$ décrit l'ensemble d'indices tels que $\sigma \prec \sigma_{i}$ et $f\left(\sigma_{i}\right)=f(\sigma)$.

3) Si $\sigma \prec \sigma^{\prime}$ et $f(\sigma)=f\left(\sigma^{\prime}\right)$, alors $A_{f}(\sigma) \supset A_{f}\left(\sigma^{\prime}\right)$.

4) $D(\sigma) \subset A_{f}(\sigma)$.

\subsubsection{Subdivision barycentrique relative.}

On sait que le morphisme $f$ n'est en général pas cellulaire pour les subdivisions barycentriques $\Delta(K)$ et $\Delta(L)$. Nous allons construire, à l'aide de 
$\triangle(K)$ et $\triangle(L)$, une subdivision relative $\triangle_{f}(K)$ telle que $f$ soit cellulaire pour $\triangle_{f}(K)$ et $\Delta(L)$. Un élément de $\Delta_{f}(K)$ est appelé simplexe relatif.

Pour cela, soit $\sigma$ une cellule de $K$ telle que $f(\sigma)=\tau$. D'abord, tout simplexe $\delta \in \Delta(K)$ contenu dans la fibre $\sigma_{\hat{\tau}}=\sigma \cap f^{-1}(\hat{\tau})$ est élément de $\triangle_{f}(K)$. Un tel élément est de type $<\hat{\sigma}_{1}, \cdots, \hat{\sigma}_{p}>$, où $f\left(\sigma_{i}\right)=\tau$ pour $1 \leqslant i \leqslant p$.

Maintenant, considérons un simplexe $\rho=<\hat{\tau}_{1}, \cdots, \hat{\tau}_{q}>$ contenu dans $\tau$. Notons $\sigma_{i j}$ la face dominante de $\sigma_{i}$ sur $\tau_{j}$. Par récurence, $\sigma_{i j_{1} \cdots j_{k}}$ est la face dominante de $\sigma_{i j_{1} \cdots j_{k-1}}$ sur $\tau_{j_{k}}$ (pour $q \geqslant j_{1}>\cdots>j_{k} \geqslant 1$ ). Notons $\delta_{\rho}$ la réunion des simplexes dont les sommets sont des centres $\hat{\sigma}_{i j_{1} \cdots j_{k}}$. Alors $\delta_{\rho}$ est un élément de $\Delta_{f}(K)$. C'est le saturé simplicial (dans $\Delta(K)$ ) de $\left\{\hat{\sigma}_{i j_{1} \cdots j_{k}}, 1 \leqslant i \leqslant p, 1 \leqslant j_{k}<\cdots<j_{1} \leqslant q\right.$, $\}$. Il est clair que l'intérieur de $\delta_{\rho}$ est homéomorphe au produit $\rho \times \delta$ et que $f\left(\delta_{\rho}\right)=\rho$. En fait, puisque $\left.f\right|_{\dot{\sigma}}$ est une fibration triviale, on a une projection $\pi: \stackrel{\circ}{\longrightarrow} \sigma_{\hat{\tau}}$ compatible avec la subdivision $\triangle(K)$. Le simplexe relatif $\delta_{\rho}$ est alors $\frac{\pi^{-1}(\delta) \cap f^{-1}(\rho)}{\pi^{\prime} \text { On }}$ notera aussi $\delta_{\rho}=<\hat{\sigma}_{1}, \cdots, \hat{\sigma}_{p} ; \hat{\tau}_{1}, \cdots, \hat{\tau}_{q}>$. Dans la figure 3 , on donne de tels exemples de subdivisions relatives.

Remarque. - Il est évident que

1) le centre relatif $C_{f}(\sigma)$ est la réunion des simplexes relatifs de forme $<\hat{\sigma} ; \hat{\tau}_{1}, \cdots, \hat{\tau}_{q}>$ où $\tau_{i} \prec \tau=f(\sigma)$ pour $1 \leqslant i \leqslant q$;

2) la cellule duale relative $A_{f}^{\prime}(\sigma)$ est la réunion de tous les simplexes relatifs de forme

$<\hat{\sigma}_{1}, \cdots, \hat{\sigma}_{p} ; \hat{\tau}_{1}, \cdots, \hat{\tau}_{q}>$ où $f\left(\sigma_{i}\right)=\tau$ pour $1 \leqslant i \leqslant p$.

2.3.5. Lemme. - 1) Notons $K_{y}$ la décomposition cellulaire de $X_{y}$ induite par $K$. Si l'on note $C_{f}^{\prime}(\sigma)=\left\{\hat{\sigma}_{y} \mid y \in \stackrel{\circ}{\tau}\right\}$ avec $\tau=f(\sigma)$, alors $C_{f}^{\prime}(\sigma)$ est l'intérieur de $C_{f}(\sigma)$ et donc $C_{f}(\sigma)=\overline{C_{f}^{\prime}(\sigma)}$.

2) Notons $D\left(\sigma_{y}\right)$ la cellule duale de $\sigma_{y}$ dans $D\left(K_{y}\right)$, alors $A_{f}(\sigma)_{y}=$ $A_{f}(\sigma) \cap f^{-1}(y)=D\left(\sigma_{y}\right)$. De plus, si $\sigma$ est de dimension relative $k$, alors $A_{f}(\sigma)$ est de dimension $p=2 m-k$, où $m$ est la dimension (complexe) de $M$.

3) $D(\sigma) \cap f^{-1}(\tau)=D\left(\sigma_{\hat{\tau}}\right)=A_{f}(\sigma)_{\hat{\tau}}$. De plus, si $\tau$ est de dimension maximale dans $Y$, alors $D(\sigma)=A_{f}(\sigma)_{\hat{\tau}}$.

Démonstration. - La partie 1) est une conséquence directe de la Remarque 2.3.4. 

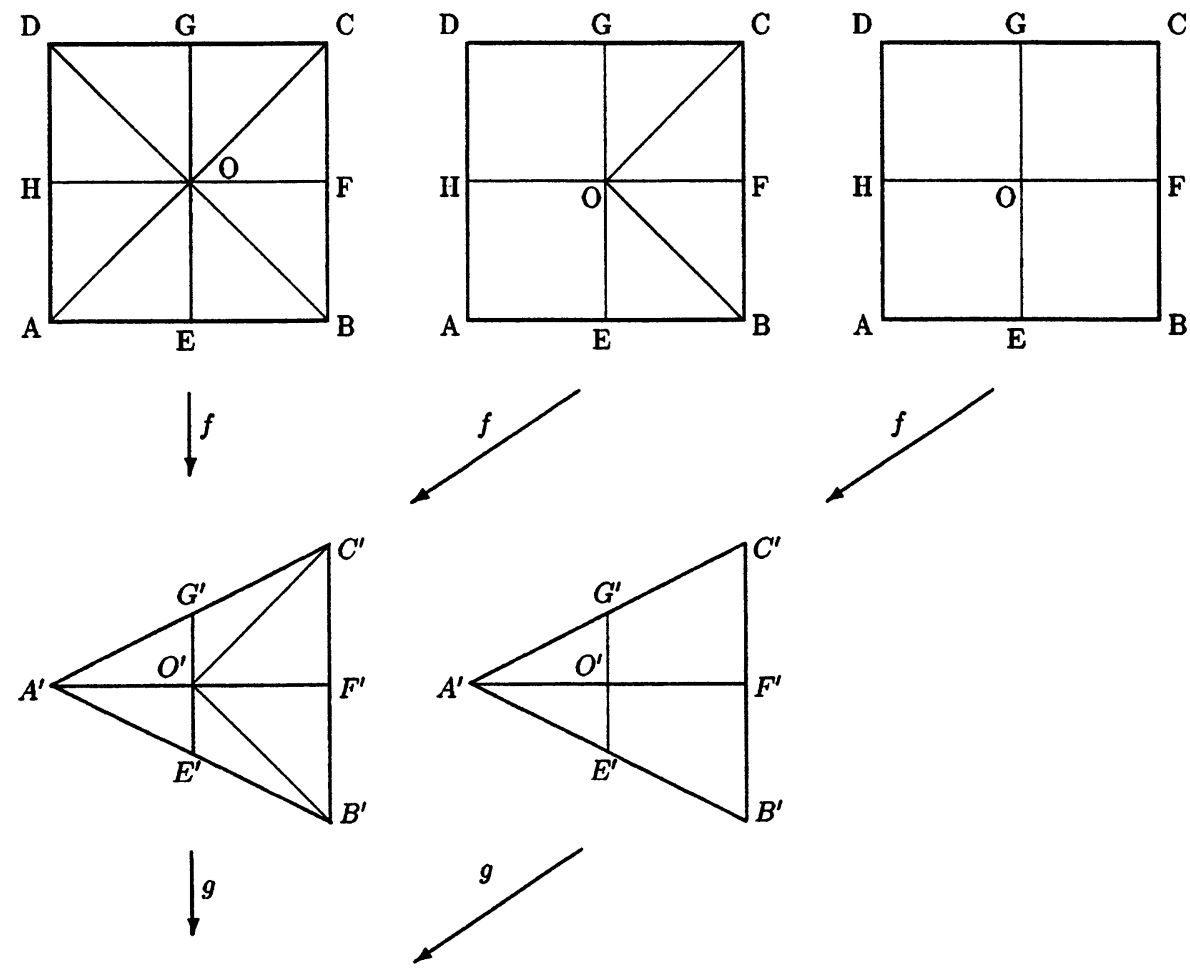

$A^{\prime \prime} Q^{\prime \prime} B^{\prime \prime}$

Fig. 3.

Les flèches verticales donnent les subdivions normales. Les flèches obliques donnent les subdivisions relatives.

2) On remarque qu'un simplexe $\delta_{y}$ de $\triangle\left(K_{y}\right)$ est la trace d'un simplexe relatif $\delta$ sur la fibre $f^{-1}(y)$. D'après la Remarque 2.3.4, on a $A_{f}(\sigma)_{y}=$ $D\left(\sigma_{y}\right)$ puisque les simplexes de $\Delta\left(K_{y}\right)$ et les simplexes de $\left.\Delta_{f}(K)\right|_{f^{-1}(y)}$ sont identiques. D'où l'assertion 2$)$.

3) D'après $\S 2.3 .4, A_{f}(\sigma)_{\hat{\tau}}=\bigcup<\hat{\sigma}_{1}, \cdots, \hat{\sigma}_{p} ; \hat{\tau}>$, est la réunion des simplexes relatifs "verticaux" sur $\tau$. Un tel simplexe relatif est évidemment contenu dans $f^{-1}(\tau)$ et dans $D(\sigma)$. Réciproquement, un simplexe $\left\langle\hat{\sigma}_{1}, \cdots, \hat{\sigma}_{p}\right\rangle$ contenu dans $D(\sigma) \cap f^{-1}(\tau)$ s'identifie à un simplexe relatif $\left\langle\hat{\sigma}_{1}, \cdots, \hat{\sigma}_{p} ; \hat{\tau}\right\rangle$. D'où l'assertion 3). 


\subsection{Composé de deux morphismes cellulaires}

2.4.1. Soit $\pi: \tilde{X} \longrightarrow X$ un morphisme analytique. Notons $g=f \circ \pi$ le morphisme composé, $\widetilde{M}$ une variété lisse dans laquelle est plongé $\widetilde{X}$ et $\widetilde{K}$ une décomposition cellulaire de $\widetilde{M}$ compatible avec $\widetilde{X}$. Supposons que le morphisme $\pi$ soit cellulaire relativement aux décompositions cellulaires $\widetilde{K}$ et $K$. Considérons une subdivision barycentrique $\Delta(\widetilde{K})$ de $\widetilde{K}$ satisfaisant les conditions de $§ 2.2 .4$.

A priori, le composé de deux morphismes cellulaires ne satisfait pas la propriété 4) de la remarque 2.2.2, i.e., si $\tau^{\prime}$ est face de $\tau=g(\tilde{\sigma})$, dans l'intersection $g^{-1}\left(\tau^{\prime}\right) \cap \tilde{\sigma}$, on peut avoir deux cellules de dimension maximale dominantes sur $\tau^{\prime}$ (voir Fig. 4). On doit dans ce cas raffiner les décompositions cellulaires pour que le composé satisfasse encore cette propriété. On peut alors construire les cellules $C_{g}(\tilde{\sigma}), A_{g}(\tilde{\sigma})$ et $B_{g}(\tilde{\sigma})$.
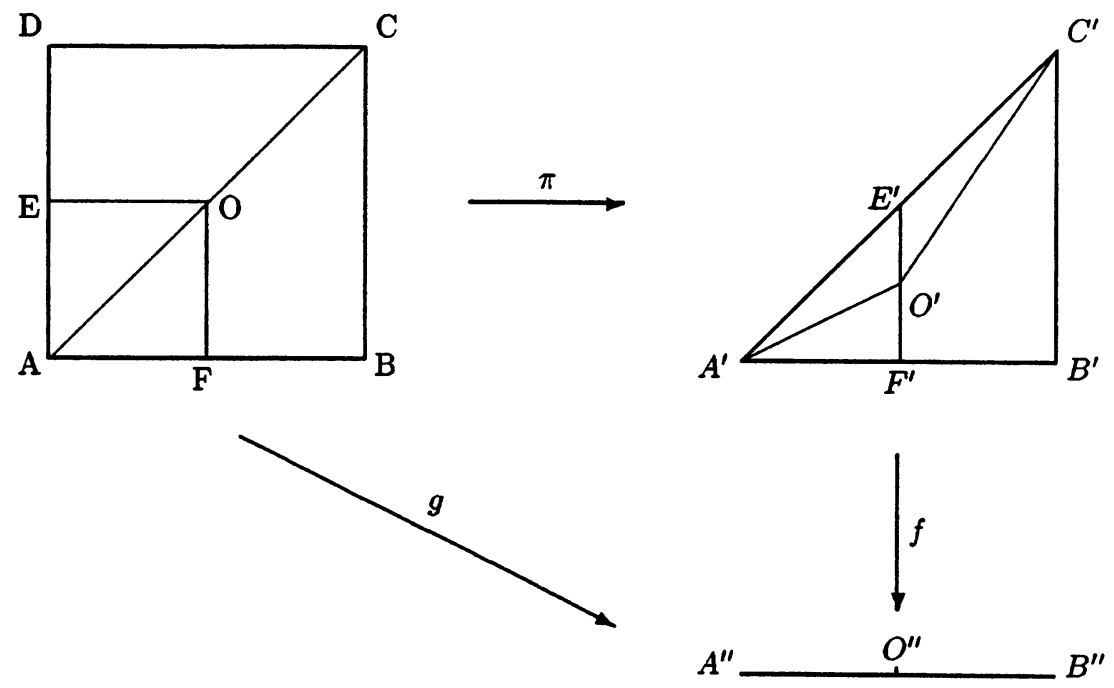

Fig. 4.

Dans cet exemple, $g^{-1}\left(B^{\prime \prime}\right)=B C \cup C D$. Il faut "couper" la cellule $A B C D$ par les cellules $A O, O C, E O, O F$ etc.

2.4.2. Lemme ([BS, Proposition 5.7]). - Pour toute cellule $\sigma$ de $K$, soit $y$ un point de l'intérieur de $\tau=f(\sigma)$. Alors $\pi^{-1}\left(D\left(\sigma_{y}\right)\right)=\bigcup D\left(\tilde{\sigma}_{y}\right)$, où $\tilde{\sigma}$ décrit l'ensemble des cellules $\pi$-horizontales de $\widetilde{K}$ contenues dans $\pi^{-1}(\sigma)$. 
2.4.3. Proposition. - Avec les hypothèses et notations de §2.4.1, pour toute cellule $\sigma$ de $K$, on a $\pi^{-1}\left(A_{f}^{\prime}(\sigma)\right)=\bigcup_{\alpha} A_{g}^{\prime}\left(\tilde{\sigma}_{\alpha}\right)$, où $\tilde{\sigma}_{\alpha}$ décrit l'ensemble des cellules $\pi$-horizontales de $\widetilde{K}$ contenues dans $\pi^{-1}(\sigma)$.

Démonstration. - C'est une conséquence directe du Lemme 2.4.2 et du Lemme 2.3.5.

\subsection{Champ relatif de repères et construction de Brasselet}

2.5.1. Reprenons les hypothèses et les notations de $\S 2.2 .4$. Soit $x$ un point dans la strate $X_{i} \subset X$, notons $T_{x}\left(\left.f\right|_{X_{i}}\right)$ l'espace tangent relatif en point $x$. Le sous-espace $T_{f}$ de $T M$ défini par la collection des $T_{x}\left(\left.f\right|_{X_{i}}\right)$ pour $x$ dans $X$ est muni d'une projection $\pi: T_{f} \longrightarrow X$. Pour un entier positif $r$, on note $T_{f}^{r}$ l'espace fibré en variétés de Stiefel des $r$-repères de $T_{f}$ sur $X$. Autrement dit, un élément de $T_{f, x}^{r}$ est un $r$-uple de vecteurs linéairement indépendants tangents à la fibre $X_{i, f(x)}$ où $X_{i}$ est la strate contenant $x$. De même, on note $T^{r} M$ l'espace fibré en variétés de Stiefel des $r$-repères de $T M$ sur $M$.

2.5.2. Notons $A$ la réunion des cellules duales relatives $A_{f}(\sigma)$ pour toutes les cellules $\sigma$ de dimension relative réelle $2(r-1)$ de $K$. Un champ de $r$-repères relatifs $\chi$ sur $A$ est une section continue de $T^{r} M$ au-dessus de $A$ telle que sa restriction à $A \cap X$ soit une section de $T_{f}^{r}$. On a construit dans [Br1, §5], un champ particulier $\chi$ de $r$-repères relatifs par la méthode de M.H. Schwartz. Ce champ est appelé champ radial relatif, il est sans singularité au-dessus des bords relatifs $B_{f}^{\prime}(\sigma)$. D'autre part, dans chaque fibre $A_{f}^{\prime}(\sigma)_{y}$ de $A_{f}^{\prime}(\sigma)$, il a au plus une singularité isolée, celle-ci étant $\hat{\sigma}_{y}$. Ses singularités sont donc situées sur la cellule $C_{f}(\sigma)$.

L'indice relatif du champ $\chi$ au point $x$ est l'indice de sa restriction à $A \cap X_{i} \cap f^{-1}(f(x))$ au point $x$. Celui-ci est défini comme dans le cas absolu (voir [BS] par exemple).

L'indice relatif du champ $\chi$ en $\hat{\sigma}_{y}$ ne dépend pas du point $y$ choisi dans l'intérieur de $f(\sigma)$ et ne dépend que de $\sigma$. On le note $\mu_{\sigma}$.

2.5.3. Considérons une cellule $\sigma$ de dimension relative $2(r-1)$ telle que $\tau=f(\sigma)$ ne soit pas de dimension maximale. Soit $\tau^{\prime}$ une cellule de $L$ admettant $\tau$ comme face. Notons $J\left(\tau^{\prime}, \sigma\right)$ l'ensemble des cellules $\sigma^{\prime}$ de dimension relative $2(r-1)$ admettant $\sigma$ comme face, et telles que $f\left(\sigma^{\prime}\right)=\tau^{\prime}$. On a le résultat suivant : 
Proposition ([Br1, Prop. 1]). - L'indice $\mu_{\sigma}$ est égal à la somme $\sum \mu_{\sigma^{\prime}}$ des indices du champ $\chi$ évalué sur toutes les cellules duales relatives $A_{f}\left(\sigma^{\prime}\right)$ $\operatorname{des} \sigma^{\prime}$ dans $J\left(\tau^{\prime}, \sigma\right)$.

2.5.4. Notons $X^{2(r-1)}$ le squelette de dimension relative $2(r-1)$ de $K$, i.e., la réunion des cellules de dimension relative $2(r-1)$. Soit $d$ la dimension relative de $X$ et posons $p=d-r+1$. Etant donnée une fonction constructible bivariante $\alpha$, supposons que la décomposition cellulaire $K$ de $X$ est $\alpha$-adaptée, c'est-à-dire que $\alpha$ est constante sur l'intérieur de chaque cellule de $K$. Posons $c(\alpha)=\sum_{\sigma} \mu_{\sigma} \alpha(\sigma) \sigma$, où la somme est prise sur toutes les cellules $\sigma$ de dimension relative 2(r-1). Dans [ $\mathrm{Br} 1$, Proposition 3] est démontré que $c(\alpha)$ est un cycle bivariant, et le théorème principal de ce travail en fournit une autre démonstration. On note encore $c(\alpha)$ la classe de ce cycle dans le groupe d'homologie bivariante $H^{-2(r-1)}\left(X^{2(r-1)} \stackrel{f}{\longrightarrow} S\right)$. Soit $i_{2(r-1)}: X^{2(r-1)} \longrightarrow X$ l'inclusion canonique, on note $c_{B}^{p}(\alpha)$ l'image directe de la classe $c(\alpha)$ dans le groupe d'homologie bivariante $H^{-2(r-1)}(X \longrightarrow S)$.

DÉfinition ([Br1]). - La classe $c_{B}^{p}(\alpha) \in H^{-2(r-1)}(X \longrightarrow S)$ est appelée classe de Chern bivariante de degré $p$ de la fonction constructible bivariante $\alpha$.

\subsection{Représentation cohomologique de la classe de Chern bivariante}

2.6.1. Fixons un plongement fermé et relatif de $X$ dans une variété lisse $S \times M$. Désormais, nous notons $K$ une décomposition cellulaire de $S \times M$, compatible avec $X$. Ainsi, les constructions seront basées sur la décomposition cellulaire $K$ de $S \times M$. Par identification de $H^{-2(r-1)}(X \longrightarrow$ $S)$ avec $H^{2(m-r+1)}(S \times M,(S \times M) \backslash X)$, le cycle $c(\alpha)$ de $\S 2.5 .4$ correspond à un cocycle $\gamma(\alpha)$ dont l'évaluation sur une fibre $A_{f}(\sigma)_{y}$ de la cellule duale relative $A_{f}(\sigma)$ est égale à :

$$
<\gamma(\alpha), A_{f}(\sigma)_{y}>=\alpha(\sigma) \mu_{\sigma} .
$$

Elle ne dépend donc pas du point $y$ choisi dans l'intérieur de $f(\sigma)$. On se permet, par abus de notation, d'écrire ce cocycle sous la forme suivante :

$$
\gamma(\alpha)=\sum_{\sigma} \alpha(\sigma) \mu_{\sigma} \delta_{b i v}\left(A_{f}(\sigma)\right),
$$

où la somme est prise sur toutes les cellules $\sigma$ de dimension relative $2(r-1)$, et $\delta_{b i v}\left(A_{f}(\sigma)\right)$ signifie la cochaîne de $C^{2(m-r+1)}(S \times M,(S \times M) \backslash X)$ prenant la valeur 1 sur chaque fibre $A_{f}(\sigma)_{y}$ pour $y$ dans l'intérieur de $f(\sigma)$. 
2.6.2. Remarquons que les cellules duales relatives $A_{f}(\sigma)$ ne forment pas une partition de $S \times M$. Cependant, les cellules $A_{f}^{\prime}(\sigma)=A_{f}(\sigma) \cap f^{-1}(\stackrel{\tau}{\tau})$ avec $\tau=f(\sigma)$ en fournissent bien une. Ce qui signifie donc que l'écriture de $\gamma(\alpha)$ a un sens, c'est-à-dire que la somme formelle ci-dessus représente bien une cochaîne de $C^{2(m-r+1)}(S \times M,(S \times M) \backslash X)$. Le lemme 4.2.3 justifiera que $\gamma(\alpha)$ est un cocycle.

LEMME. - La $p^{\text {ème }}$ classe de Chern bivariante $c_{B}^{p}(\alpha)$ est représentable par le cocycle $\gamma(\alpha)$.

\subsection{Relèvement du champ de repères relatif}

Dans ce paragraphe, on va relever le champ de $r$-repères relatifs défini au-dessus de $A \subset X$ en $r$ sections linéairement indépendantes du fibré tautologique $E^{t}$ de $G_{t}\left(\left.f\right|_{X}\right) \stackrel{\pi}{\longrightarrow} X$ au-dessus de $\pi^{-1}(A)$ (cf. $\S 1.3 .1$ ).

2.7.1. Remarquons que le fibré $E^{t}$ est un sous fibré du fibré $\left.\pi^{*} T M\right|_{X}$, c'est-à-dire du produit fibré $G_{t}\left(\left.f\right|_{X}\right) \times_{X}\left(\left.T M\right|_{X}\right)$. On a une projection naturelle $\pi_{*}:\left.E^{t} \longrightarrow T M\right|_{X}$, définie par $\pi_{*}(\tilde{x}, v)=v$, où $\tilde{x} \in G_{t}\left(\left.f\right|_{X}\right)$, $x=\pi(\tilde{x})$ et $v \in T_{x} M$. On suppose que le sous-espace $T_{f}$ de $T M$ est obtenu à partir d'une bonne stratification (voir §1.4.3). D'après la Remarque 1.4.5, si l'espace but est une courbe lisse, on peut toujours avoir une bonne stratification.

Proposition. - Avec les hypothèses et les notations ci-dessus, on a

a) Etant donnés $x \in X$ et $\chi(x) \in T_{f}(x)$, pour tout point $\tilde{x} \in \pi^{-1}(x)$, il existe un unique vecteur $\tilde{\chi}(\tilde{x})$ de $E_{\tilde{x}}^{t}$ tel que $\pi_{*}(\tilde{\chi}(\tilde{x}))=\chi(x)$.

b) Si $\chi$ est une section de $T_{f}$ au-dessus d'une partie $A$ de $X$, alors $\tilde{\chi}$ définit une section de $E^{t}$ au-dessus de $\widetilde{A}=\pi^{-1}(A)$.

Démonstration.

a) Considérons le produit fibré $G_{t}\left(\left.f\right|_{X}\right) \times_{X} T_{f}$ de $p: T_{f} \longrightarrow X$ et de $\pi: G_{t}\left(\left.f\right|_{X}\right) \longrightarrow X$. L'espace $T_{f}$ étant plongé dans $\left.T M\right|_{X}$, pour montrer la proposition, il suffit de montrer que $G_{t}\left(\left.f\right|_{X}\right) \times_{X} T_{f}$ est plongé dans $E^{t} . \mathrm{Si}$ c'est le cas, on a un diagramme commutatif :

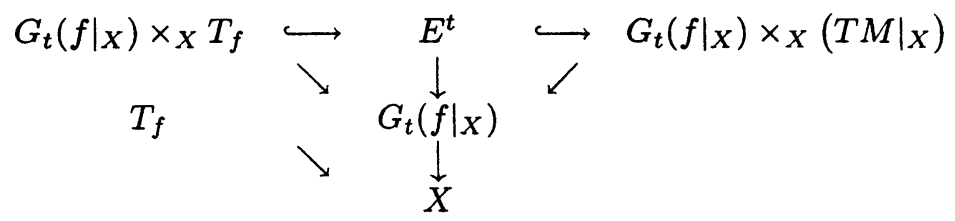


et le relèvement est naturellement défini par le produit fibré, i.e. par $\tilde{\chi}(\tilde{x})=$ $(\tilde{x}, \chi(x))$.

Pour tout point $(\tilde{x}, v)$ de $G_{t}\left(\left.f\right|_{X}\right) \times_{X} T_{f}$, supposons que $x=\pi(\tilde{x})$ soit dans la strate $X_{i}$, le vecteur $v$ est donc dans l'espace tangent relatif $T_{x}\left(\left.f\right|_{X_{i}}\right)$. Soit $X^{0}$ la strate dense de $X$ sur laquelle $f$ est de co-rang maximum. Par définition de $G_{t}\left(\left.f\right|_{X}\right)$, le point $\tilde{x}$ est une paire $(x, H)$ telle qu'il existe une suite de points de $X^{0}$ tendant vers $x$ et que $H$ soit la limite des espaces tangents relatifs $T_{x_{k}}\left(\left.f\right|_{X^{0}}\right)$ quand $x_{k}$ tend vers $x$. D'après la condition $A_{f}$ de Thom, on a $T_{x}\left(\left.f\right|_{X_{i}}\right) \subset H$ et donc $v \in H$. Ceci montre que le point $(\tilde{x}, v)$ est dans $E^{t}$, d'où, $G_{t}\left(\left.f\right|_{X}\right) \times_{X} T_{f} \subset E^{t}$.

b) Evidemment, si $\chi$ est un champ continu, alors la section $\tilde{\chi}$ construite comme ci-dessus est aussi un champ continu.

\subsection{Classe fondamentale bivariante}

Dans ce paragraphe, on explicite un cocycle de la classe fondamentale bivariante.

2.8.1. Définition. - Soit $f: X \longrightarrow S$ un morphisme analytique de dimension relative $d$. La classe fondamentale bivariante de $X$ est la classe $[X]_{f}$ en homologie bivariante $H^{-2 d}(X \stackrel{f}{\longrightarrow} S)$ telle que, pour tout point $y$ de $S$, l'image réciproque $j_{y}^{*}\left([X]_{f}\right)$ par l'inclusion $j_{y}:\{y\} \rightarrow S$ soit la classe fondamentale de la fibre $X_{y}$ dans $H_{2 d}\left(X_{y}\right)$.

Cette notion de classe fondamentale bivariante a été introduite par J.-L. Verdier ([Ve2]), D. Barlet([Ba2]) et M. Kaddar([Ka]) sous le nom de "classe fondamentale relative".

2.8.2. Remarquons que tout morphisme analytique propre surjectif $f$ : $X \longrightarrow S$ avec $X$ irréductible et $S$ une courbe lisse est plat ([Hi1]). En particulier les morphismes $\left.f\right|_{Z}: Z \longrightarrow S$ et $f \circ \nu_{f}: N_{f}(Z) \longrightarrow S$ sont plats, où $Z$ est un cycle de $X$ tel que la restriction du morphisme $f$ sur chaque composante irréductible de $Z$ soit ouverte (ainsi, $Z$ est un cycle bivariant d'après Sabbah, voir §3.1.1). D'après les théorèmes de [Ve2] ou [Ba2], leur classe fondamentale bivariante sur $S$ est bien définie.

2.8.3. On peut aussi voir cette classe fondamentale bivariante de la façon suivante : avec les notations de $\S 2.6 .1$, considérons la cochaîne $\Gamma=$ $\sum \delta(D(\sigma))$, où la somme est prise sur toutes les cellules $\sigma$ de dimension maximale dans $X$, et où $\delta(D(\sigma))$ désigne la cochaîne élémentaire associée à la cellule duale $D(\sigma)$. La cochaîne $\Gamma$ est un cocycle dont on note $[\Gamma]$ la classe dans $H^{2(m-d)}(S \times M,(S \times M) \backslash X)=H^{-2 d}(X \stackrel{f}{\longrightarrow} S)$. 
THÉORÈME. - Soit $f: X \longrightarrow S$ un morphisme analytique propre de dimension relative $d$, où $S$ est une courbe lisse. Alors la classe $[\Gamma] \in H^{-2 d}(X \stackrel{f}{\longrightarrow} S)$ est la classe fondamentale bivariante $[X]_{f}$.

Démonstration. - Nous montrons d'abord qu'il existe un ouvert dense $U$ de $S$, tel que si $j_{y}$ désigne l'inclusion d'un point $y$ de $U$ dans $S$, alors l'image réciproque $j_{y}^{*}[\Gamma]$ est la classe fondamentale de $X_{y}$.

Prenons pour $U$ la réunion de toutes les cellules ouvertes de dimension maximale de $L$, c'est un ouvert dense de $S$. Remarquons que pour tout point $y$ de $U$, la décomposition $K$ induit une décomposition cellulaire $K_{y}$ de $\{y\} \times M$ compatible avec $X_{y}$. D'après le Lemme 2.3.5, les cellules duales $D\left(\sigma_{y}\right)=A_{f}(\sigma)_{y}$ de $\sigma_{y}$ dans $\{y\} \times M$, pour tous les points $y$ à l'intérieur de $\tau=f(\sigma)$, forment une famille $\mathcal{D}$ de cellules, toutes homéomorphes à $D(\sigma)=D\left(\sigma_{\hat{\tau}}\right)$. Pour tout point $y$ de $U$, le cocycle $\Gamma$ induit donc un cocycle $\Gamma_{y}=\sum \delta\left(D\left(\sigma_{y}\right)\right)$ de $X_{y}$, où la somme est prise sur l'ensemble des cellules $\sigma$ telles que $X_{y} \cap \sigma=\sigma_{y}$ soit non vide (de dimension maximale dans $X_{y}$ ). La classe du cocycle $\Gamma_{y}$ est la classe fondamentale de $X_{y}$ dans $H^{2(m-d)}(\{y\} \times$ $\left.M,\{y\} \times M \backslash X_{y}\right) \simeq H_{2 d}\left(X_{y}\right)$, par isomorphisme d'Alexander ([Br2]).

Notons $T$ un voisinage tubulaire de $X$ dans $S \times M$ formé des cellules fermées $\sigma$ tels que $\sigma \cap X \neq \emptyset$. Alors

$$
[X]_{f} \in H^{2(m-d)}(S \times M, S \times M \backslash X) \simeq H^{2(m-d)}(T, T \backslash X) \simeq H^{2(m-d)}(T, \partial T),
$$

par excision et rétraction de $T \backslash X$ sur $\partial T$.

Dans ce dernier groupe de cohomologie, $[X]_{f}$ est représentable par un cocycle $\Gamma^{\prime}=\sum n_{\sigma} \delta(D(\sigma))$, où $\operatorname{dim}(D(\sigma))=2(m-d)$, c'est-à-dire où $\sigma$ décrit les cellules de dimension maximale de $X$.

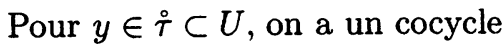

$$
j_{y}^{*}\left(\Gamma^{\prime}\right)=j_{y}^{*}\left(\sum_{\sigma} n_{\sigma} \delta(D(\sigma))\right)=\sum_{f(\sigma)=\tau} n_{\sigma} \delta\left(D\left(\sigma_{y}\right)\right) .
$$

Par isomorphisme d'Alexander écrit pour $X_{y} \subset\{y\} \times M$, on obtient un cycle $\sum_{\sigma} n_{\sigma} \sigma_{y}$, où $\sigma$ décrit les cellules de dimension maximale de $X$ telles que $\underset{\sigma}{f}(\sigma)=\tau$. Par définition de $[X]_{f}$, ce cycle est un représentant de la classe fondamentale de $X_{y}$. Or, cette dernière est représentable par un unique cycle en homologie cellulaire $H_{2 d}\left(K_{y}\right)$, et celui-ci s'écrit $\sum \sigma_{y}$, où la somme porte sur toutes les cellules $\sigma_{y}$ de dimension maximale dans $X_{y}$. On en déduit $n_{\sigma}=1$ pour toute cellule $\sigma$, donc $\Gamma^{\prime}=\Gamma$. 


\section{Construction de Sabbah}

On rappelle maintenant les constructions et résultats de C. Sabbah.

\subsection{Cycles bivariants}

3.1.1. Définition ([Sab2], [Sab3]). - Soit $f: X \longrightarrow S$ un morphisme analytique propre avec $S$ une courbe lisse. Soit $Z=\sum n_{i} Z_{i}$ un cycle de $X$, où $Z_{i}$ est un sous-espace analytique fermé irréductible de $X$ pour tout $i$. On dit que $Z$ est un cycle bivariant si, pour toute composante $Z_{i}$ de $Z$, la restriction $\left.f\right|_{Z_{i}}$ est un morphisme ouvert.

On note $\left.f\right|_{Z}$ ou simplement $Z$ le cycle bivariant associé, et $\mathcal{Z}(X \stackrel{f}{\longrightarrow} S)$ le groupe abélien libre des cycles bivariants.

Une définition des cycles bivariants pour un morphisme analytique propre est donnée à l'aide de l'espace conormal relatif ([Sab3]). Elle est compatible avec celle donnée ci-dessus dans le cas où l'espace but est une courbe lisse.

Dans toute la suite, les constructions seront faites sur les éléments de la base canonique du Z-module libre $\mathcal{Z}(X \stackrel{f}{\longrightarrow} S)$. Les constructions ainsi effectuées s'étendent par linéarité.

3.1.2. Rappelons la définition de la spécialisation des cycles. Soit $j_{y}$ : $y \longrightarrow S$ l'inclusion d'un point $y$ de $S$. Soit $\left.f\right|_{z}$ un cycle bivariant. Le morphisme $\left.f\right|_{Z}$ étant surjectif sur $S$, est sans éclatement en codimension 0 . D'après le lemme 1.4.4, toute composante irréductible de l'espace $C\left(\left.f\right|_{z}, M\right)_{y}$ est l'espace conormal d'un sous-espace analytique de $X_{y}$. On associe alors à $\left.f\right|_{z}$ le cycle $j_{y}^{*}\left(\left.f\right|_{z}\right)$ de $X_{y}$ tel que l'on ait $C\left(j_{y}^{*}\left(\left.f\right|_{z}\right), M\right)=C\left(\left.f\right|_{z}, M\right)_{y}$. On appelle $j_{y}^{*}\left(\left.f\right|_{Z}\right)$ la spécialisation de $\left.f\right|_{Z}$ au dessus de $y$.

\subsection{Obstruction d'Euler locale relative}

3.2.1. La notion d'obstruction d'Euler locale relative, dûe à Sabbah [Sab3], est définie comme suit.

DÉFINITION ([Sab3, §3]). - Soit $\left.f\right|_{Z}$ un cycle bivariant, pour tout point $x \in|Z|$, on définit l'obstruction d'Euler locale relative $E u_{f}(Z, x)$ de $Z$ au point $x$ comme étant l'obstruction d'Euler locale $E u\left(j_{f(x)}^{*}\left(\left.f\right|_{Z}\right), x\right)$.

L'obstruction d'Euler locale relative duale est définie par

$$
\begin{aligned}
E \check{u}_{f}(Z, x)= & (-1)^{\operatorname{dim}_{f}(Z)} E u_{f}(Z, x) . \\
& -181-
\end{aligned}
$$


3.2.2. Théorème. ([Sab3, Th.4.1]) Pour tout cycle bivariant $\left.f\right|_{z}$, l'obstruction d'Euler locale relative $E u_{f}(Z, \cdot)$ est une fonction constructible bivariante. On définit ainsi une transformation de Grothendieck entre la théorie des cycles bivariants $\mathcal{Z}(X \longrightarrow S)$ et la théorie des fonctions constructibles bivariantes $\mathcal{F}(X \longrightarrow S)$. C'est un isomorphisme.

\subsection{Classe de Chern bivariante de Sabbah}

3.3.1. Soit $Z$ un cycle bivariant irréductible et réduit. Notons $d$ sa dimension relative. Pour tout entier $p$ compris entre 0 et $d$, on pose $q=d-p$. Soit $\nu_{f}: N_{f}(Z) \longrightarrow Z$ la transformation de Nash relative, et notons $g$ le morphisme composé $f \circ \nu_{f}$. La classe fondamentale bivariante $\left[N_{f}(Z)\right]_{g}$ existe dans $H^{-2 d}\left(N_{f}(Z) \stackrel{g}{\longrightarrow} S\right.$ ) (cf. $\left.\S 2.8 .2\right)$. D'autre part, soit $\theta$ le fibré de Nash relatif au-dessus de $N_{f}(Z)$, la classe de Chern $c^{q}(\theta)$ est une classe dans $H^{2 q}\left(N_{f}(Z) \stackrel{i d}{\longrightarrow} N_{f}(Z)\right)$. En utilisant le produit bivariant,

$$
H^{2 q}\left(N_{f}(Z) \stackrel{i d}{\longrightarrow} N_{f}(Z)\right) \otimes H^{-2 d}\left(N_{f}(Z) \stackrel{g}{\longrightarrow} S\right) \longrightarrow H^{-2 p}\left(N_{f}(Z) \stackrel{g}{\longrightarrow} S\right),
$$

on a $c^{q}(\theta) \cdot\left[N_{f}(Z)\right]_{g} \in H^{-2 p}\left(N_{f}(Z) \stackrel{g}{\longrightarrow} S\right)$. Notons Inc $: Z \longrightarrow X$ l'inclusion, on obtient une classe $c_{S}^{p}(Z):=\operatorname{Inc} c_{*} \nu_{f *}\left(c^{q}(\theta) \cdot\left[N_{f}(Z)\right]_{g}\right)$ dans $H^{-2 p}(X \stackrel{f}{\longrightarrow} S)$.

3.3.2. On remarque que, pour tout point $y \in S$, on obtient par image réciproque une classe $j_{y}^{*}\left(c_{S}^{p}(Z)\right)$ dans $H^{-2 p}\left(X_{y} \stackrel{f}{\longrightarrow} y\right)=H_{2 p}\left(X_{y}\right)$. Cette classe n'est autre que la classe de Chern-Mather $c_{p}\left(\left.j_{y}^{*} f\right|_{z}\right)$.

3.3.3. Définition. ([Sab2], [Sab3]) Soit $f: X \longrightarrow S$ un morphisme analytique propre, soit $Z$ un cycle bivariant. La $p^{\grave{e} m e}$ classe de Chern bivariante de $Z$ est la classe bivariante $c_{S}^{p}(Z)$ dans $H^{-2 p}(X \longrightarrow S)$. La $p^{\text {ème }}$ classe de Chern bivariante $c_{S}^{p}(\alpha)$ d'une fonction constructible bivariante $\alpha$ est la classe $c_{S}^{p}\left(E u_{f}^{-1}(\alpha)\right)$.

\section{Coïncidence des deux constructions}

Dans toute la suite, on considère un morphisme analytique $f: X \longrightarrow S$ où $S$ est une courbe lisse. Fixons un plongement relatif de $X$ dans une variété analytique $M$, et prenons les hypothèses et les notations de $§ 2.6 .1$.

\subsection{Obstruction d'un champ de repères et celle de son relevé}

Soit $Z$ un cycle bivariant dans $\mathcal{Z}(X \stackrel{f}{\longrightarrow} S)$. On établit dans ce paragraphe, une relation entre l'indice d'un champ de repères relatif et l'obstruction de son relevé sur $N_{f}(Z)$ établissant ainsi un résultat analogue à celui 
de $[\mathrm{BS}]$. Pour cela, on considère la définition topologique de l'obstruction d'Euler locale relative comme dans [Sab2, §4.7] à l'aide de l'espace conormal relatif.

4.1.1. On fixe une métrique hermitienne $C^{\infty}$ sur $M$. Considérons une boule $B_{\varepsilon}(x)$ assez petite centrée en $x$ et posons $U=\tau_{f}^{-1}\left(B_{\varepsilon}(x) \cap Z_{f(x)}\right)$ dans $C\left(j_{f(x)}^{*}\left(\left.f\right|_{Z}\right), M\right)$. On remarque que, comme le morphisme $\tau_{f}$ est analytique, le bord $\partial U$ de $U$ est l'image inverse par $\tau_{f}$ du bord $\partial B_{\varepsilon}(x) \cap Z_{f(x)}$. Choisissons de plus la boule $B_{\varepsilon}(x)$ géodésique. On peut définir un champ $C^{\infty}$ réel de vecteurs sortants sur $B_{\varepsilon}(x) \backslash\{x\}$ par $v(y)=\overrightarrow{x y}$, où $y$ est un point quelconque de $B_{\varepsilon}(x) \backslash\{x\}$ et $\overrightarrow{x y}$ est le vecteur dans l'espace tangent réel $T_{y} M$. Pour $\varepsilon$ assez petit, la restriction de ce champ à $B_{\varepsilon}(x) \cap Z_{f(x)}$ se projette sur $T\left(\left.f\right|_{z}\right)_{f(x)}$ (la collection des espaces tangents aux strates de $Z_{f(x)}$ ). Notons $\vartheta$ l'image de cette projection. Elle est non nulle sur $B_{\varepsilon}(x) \backslash\{x\}$. On peut relever $\vartheta$ sur $U$ comme section du fibré réel sous-jacent de $E$ (qu'on note encore $E$ ) non nulle sur $\partial U$. On obtient une section $s:(U, \partial U) \longrightarrow\left(E, E^{*}\right)$, où $E^{*}$ désigne le sous-espace des vecteurs non-nuls de $E$. Soit $\omega_{E}$ la classe de Thom de la paire de fibrés $\left(E, E^{*}\right)$, i.e. la classe d'obstruction fondamentale de $\left(E, E^{*}\right)$ dans $H^{2(m-1)}\left(E, E^{*}\right)$, on obtient une classe $s^{*} \omega_{E}$ de $H^{2(m-1)}(U, \partial U)$. L'évaluation de cette classe sur la classe fondamentale $[U, \partial U]$ est un entier égal à $(-1)^{m-1} E \check{u}\left(j_{f(x)}^{*}(f \mid z), x\right)$, (cf. [Sab2]).

4.1.2. On peut obtenir l'obstruction d'Euler locale relative via le transformé de Nash relatif de façon analogue à [BS]. Cela n'est pas trivial car la fibre du transformé de Nash relatif $N_{f}(Z)_{y}$ peut être différente du transformé de Nash de la fibre spécialisée $N\left(j_{y}^{*}\left(\left.f\right|_{Z}\right)\right)$.

Pour cela, on reprend les notations de $\S 4.1 .1$, notons $V=\nu_{f}^{-1}\left(B_{\varepsilon}(x) \cap\right.$ $\left.Z_{f(x)}\right)$ et son bord $\partial V=\nu_{f}^{-1}\left(\partial B_{\varepsilon}(x) \cap Z_{f(x)}\right)$ dans $N_{f}(Z)$. La projection $\vartheta$ du champ radial sur $T\left(\left.f\right|_{Z}\right)_{f(x)}$ peut aussi être relevée au dessus de $V$ comme section du fibré de Nash relatif $\theta$ réel sous-jacent, non nulle sur $\partial V$. On obtient alors une section $t:(V, \partial V) \longrightarrow\left(\theta, \theta^{*}\right)$. Soit $\omega_{\theta}$ la classe de Thom associée au fibré $\theta$, on obtient une classe $t^{*} \omega_{\theta}$ de $H^{2 d}(V, \partial V)$.

4.1.3. Proposition. - La classe d'obstruction $t^{*} \omega_{\theta}$ évaluée sur la classe fondamentale $[V, \partial V]$ est un entier égal à $E u_{f}(Z, x)$.

Démonstration. - On considère le diagramme cartésien suivant :

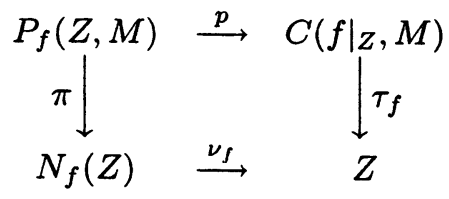


L'espace $P_{f}(Z, M)$ est aussi le projectifié de l'espace total du fibré $F$ noyau de $\nu_{f}^{*} T^{*} M \longrightarrow \theta^{-} \longrightarrow 0$.

Sur $P_{f}(Z, M)$, on a deux fibrés image réciproque : $\pi^{*} \theta$ et $p^{*} E$. Notons $(\widetilde{U}, \partial \widetilde{U})=p^{-1}(U, \partial U)=\pi^{-1}(V, \partial V)$. Comme le problème d'obstruction est local, on peut identifier la variété $M$ avec $\mathbf{C}^{m}$ et identifier sur $\widetilde{U}$ le fibré $p^{*} E$ avec le sous-fibré en hyperplans du fibré trivial $\widetilde{U} \times \mathbf{C}^{m}$. Le fibré $\pi^{*} \theta$ est alors naturellement un sous fibré de $p^{*} E$.

Le fibré tautologique $\mathcal{O}(-1)$ étant naturellement sous-fibré de $\pi^{*} F$ et de $\pi^{*} \nu_{f}^{*} T^{*} M$, le quotient $\pi^{*} \nu_{f}^{*} T^{*} M / \mathcal{O}(-1)$ coïncide avec $p^{*} E^{-}$. En prenant les quotients des fibrés dans la suite exacte de fibrés sur $\widetilde{U}$ :

$$
0 \longrightarrow \pi^{*} F \longrightarrow \pi^{*} \nu_{f}^{*} T^{*} M \longrightarrow \pi^{*} \theta^{-} \longrightarrow 0,
$$

on obtient une suite exacte

$$
0 \longrightarrow \pi^{*} F / \mathcal{O}(-1) \longrightarrow p^{*} E^{-} \longrightarrow \pi^{*} \theta^{-} \longrightarrow 0 .
$$

Par dualisation on a une suite exacte de fibrés :

$$
0 \longrightarrow \pi^{*} \theta \stackrel{\iota}{\longrightarrow} p^{*} E \longrightarrow V_{\check{F}} \longrightarrow 0 .
$$

où $V_{\breve{F}}$ est le fibré dual $\left[\pi^{*} F / \mathcal{O}(-1)\right]^{`}$, de rang $m-d-1$.

D'une part, on a deux relevés de sections sur $(\widetilde{U}, \partial \widetilde{U})$, l'un est le relevé $s \circ p$ de la section $s$ par $p$ comme section du fibré $p^{*} E$; l'autre est le relevé $t \circ \pi$ de la section $t$ par $\pi$ comme section du fibré $\pi^{*} \theta$. On remarque que $s \circ p$ coïncide avec l'image par l'inclusion $\iota$ de $t \circ \pi$.

D'autre part, on a deux classes d'obstruction. L'une $p^{*} s^{*} \omega_{E} \in H^{2(m-1)}$ $(\widetilde{U}, \partial \widetilde{U})$ est la classe d'obstruction à étendre $s \circ p$ à $\widetilde{U}$ et l'autre $\pi^{*} t^{*} \omega_{\theta} \in$ $H^{2 d}(\widetilde{U}, \partial \widetilde{U})$ est la classe d'obstruction à étendre $t \circ \pi$ à $\widetilde{U}$.

On utilise alors la formule de classes d'obstructions pour une suite exacte de fibrés, (voir [GS, §4.2] par exemple). En remarquant que la classe d'Euler du fibré $V_{F}$ est la classe de Chern de degré maximal $c^{m-d-1}\left(V_{\tilde{F}}\right)$, on obtient

$$
p^{*} s^{*} \omega_{E}=\pi^{*} t^{*} \omega_{\theta} \cup c^{m-d-1}\left(V_{\check{F}}\right) .
$$

Or, on a les égalités suivantes :

$$
\begin{gathered}
p^{*} s^{*} \omega_{E} \cdot[\widetilde{U}]=s^{*} \omega_{E} \cdot[U]=(-1)^{m-1} E \check{u}_{f}(Z, x) . \\
\left.\pi_{*}\left(\pi^{*} t^{*} \omega_{\theta} \cup c^{m-d-1}\left(V_{\check{F}}\right)\right) \cdot[\widetilde{U}]\right)=(-1)^{m-d-1} t^{*} \omega_{\theta} \cdot[V], \\
-184-
\end{gathered}
$$


où la dernière égalité vient du résultat suivant (voir [Sab4] par exemple):

Soit $F$ un fibré vectoriel complexe de rang $n$ sur $X$, et $\mathbf{P}(F)$ le projectifié de $F$. Si $\pi: \mathbf{P}(F) \longrightarrow X$ désigne la projection, on a une inclusion naturelle du fibré tautologique $\mathcal{O}(-1)$ dans $\pi^{*} F$. On note $V_{F}$ le fibré quotient et $V_{\tilde{F}}$ son dual, on a alors dans $H_{*}(X, \mathbf{Z})$ les égalités :

$$
\begin{gathered}
\pi_{*}\left(c^{n-1}\left(V_{F}\right) \cap[\mathbf{P}(F)]\right)=[X] . \\
\pi_{*}\left(c^{n-1}\left(V_{\breve{F}}\right) \cap[\mathbf{P}(F)]\right)=(-1)^{n-1}[X] .
\end{gathered}
$$

Les égalités (1), (2) et (3) nous donnent $(-1)^{m-d-1} t^{*} \omega_{\theta} \cdot[V]=(-1)^{m-1}$ $E \check{u}_{f}(Z, x)$, donc $t^{*} \omega_{\theta} \cdot[V]=E u_{f}(Z, x)$, en rappelant que $E \check{u}_{f}(Z, x)=$ $(-1)^{d} E u_{f}(Z, x)$.

4.1.4. Relation entre obstruction d'un champ de repères relatifs et celle de son relevé sur $N_{f}(X)$.

La variété $S \times M$ étant munie d'une décomposition cellulaire $K$ compatible avec $X$, comme dans $\S 2.5 .2$, on note $A$ la réunion des cellules duales relatives $A_{f}(\sigma)$ des cellules $\sigma$ de dimension relative $2(r-1)$. La trace $A \cap X$ est de dimension relative $2 q=2(d-r+1)$. Soit $\chi^{r}$ un champ radial de $r$-repères relatifs défini sur $A$. Ce champ n'est pas à singularités isolées, cependant sa restriction à chaque fibre l'est. Supposons $x \in A$, on note $I\left(\chi^{r}, x\right)$ l'indice en $x$ de la restriction de $\chi^{r}$ à la fibre contenant $x$ (cet indice est défini comme dans [BS], en tant qu'indice d'un champ radial sur un ensemble stratifié). On utilise la proposition 2.7.1 pour relever ce champ radial en une section $\tilde{\chi}^{r}$ du fibré $\theta^{r}$ (dont la fibre est la variété de Stiefel $W_{d, r}$ des $r$-repères de l'espace $\mathbf{C}^{d}$ ) au dessus de

$$
V^{\prime}=\nu_{f}^{-1}\left(B_{\varepsilon}(x) \cap A \cap f^{-1}(f(x))\right) .
$$

Cet espace est de dimension réelle $2 q$.

La section $\tilde{\chi}^{r}$ n'a pas de singularité sur le bord de $V^{\prime}$

$$
\partial V^{\prime}=\nu_{f}^{-1}\left(\partial B_{\varepsilon}(x) \cap A \cap f^{-1}(f(x))\right) .
$$

Par construction du champ radial, les singularités de $\chi^{r}$ sont seulement des zéros du dernier vecteur $v^{r}$, c'est-à-dire que les $r-1$ premiers vecteurs $\chi^{r-1}$ de $\chi^{r}$ forment un champ de $(r-1)$-repères sans singularités. Les relèvements $\tilde{\chi}^{r-1}$ sont linéairement indépendants sur $V^{\prime}$. Ils engendrent donc un sous-fibré de rang $r-1$ du fibré $\theta$ au-dessus de $V^{\prime}$. Notons $\theta^{\prime}$ le quotient de $\theta$ par ce sous-fibré. Il est de rang $d-r+1$. La dernière section $\tilde{v}^{r}$ 
détermine une section de $\theta^{\prime}$. Soit $\omega_{\theta^{\prime}}$ la classe de Thom associé au fibré $\theta^{\prime}$, on obtient ainsi une classe $\tilde{v}^{r *} \omega_{\theta^{\prime}} \in H^{2(d-r+1)}\left(V^{\prime}, \partial V^{\prime}\right)$, dont l'évaluation sur la classe fondamentale $\left[V^{\prime}, \partial V^{\prime}\right]$ est un entier noté $O b s\left(\widetilde{\chi}^{r}, V^{\prime}\right)$.

4.1.5. Théorème. $-\operatorname{Obs}\left(\widetilde{\chi}^{r}, V^{\prime}\right)=I\left(\chi^{r}, x\right) \cdot E u_{f}(Z, x)$.

Etudions d'abord le cas absolu (le cas où le morphisme $f$ est constant).

Soit $Z$ un ensemble analytique de dimension $d$ dans $M$ muni d'une décomposition cellulaire. On note $\tau: C(Z, M) \longrightarrow Z$ la projection de l'espace conormal sur $Z$ et le fibré en hyperplans de base $C(Z, M)$. Notons $\widehat{\chi}^{r}$ le relèvement d'un champ radial $\chi^{r}$ comme section du fibré $E^{r}$ associé à $E$ au-dessus de $U=\tau^{-1}\left(A \cap B_{\varepsilon}(x)\right)$. Le champ est sans singularité sur le bord de $A \cap B_{\varepsilon}(x)$. Le relèvement $\widehat{\chi}^{r}$ est alors sans singularité sur le bord $\partial U$. L'évaluation sur $[U, \partial U]$ de la classe d'obstruction à étendre $\widehat{\chi}^{r}$ à l'intérieur de $U$ est un entier noté $\operatorname{Obs}\left(\widehat{\chi}^{r}, U\right)$.

4.1.6. Lemme. $-O b s\left(\widehat{\chi}^{r}, U\right)=(-1)^{m-1} I\left(\chi^{r}, x\right) \cdot E \check{u}(Z, x)$.

Démonstration. On utilisera les notations suivantes : $\theta^{\prime}$ (resp. $E^{\prime}$ ) est le quotient du fibré de Nash $\theta$ (resp. $E$ ) par le sous-fibré engendré par les $r-1$ premières sections de $\tilde{\chi}^{r}$ (resp. $\widehat{\chi}^{r}$ ) au dessus de $V=\nu^{-1}\left(A \cap B_{\varepsilon}(x)\right)$ (resp. U).

On considère le produit fibré :

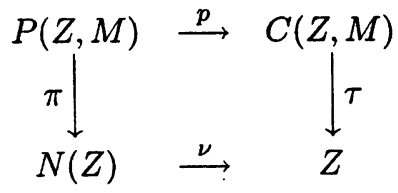

Sur $P(Z, M)$, on a une inclusion de $\pi^{*} \theta^{\prime} \longleftrightarrow p^{*} E^{\prime}$, d'où la suite exacte

$$
0 \longrightarrow \pi^{*} \theta^{\prime} \iota p^{*} E^{\prime} \longrightarrow V_{F}^{\prime-} \longrightarrow 0 .
$$

On remarque que le fibré $V_{F}^{\prime-}$ est isomorphe au fibré dual $V_{F}^{-}$, de rang $m-d-1$, puisque la suite exacte provient de la suite exacte

$$
0 \longrightarrow \pi^{*} \theta \stackrel{\iota}{\longrightarrow} p^{*} E \longrightarrow V_{\bar{F}} \longrightarrow 0,
$$

et passant aux quotients par le relevé du sous-fibré engendré par les $\chi^{r-1}$.

En appliquant les arguments de la démonstration de la proposition 4.1.3 aux fibrés $\pi^{*} \theta^{\prime}$ et $p^{*} E^{\prime}$, ainsi qu'à leurs sections, on obtient l'égalité :

$$
\begin{aligned}
\operatorname{Obs}\left(\widehat{\chi}^{r}, U\right)= & (-1)^{m-d-1} \operatorname{Obs}\left(\tilde{\chi}^{r}, V\right) . \\
& -186-
\end{aligned}
$$


D'après [BS, Th.11.1], on obtient

$\operatorname{Obs}\left(\widehat{\chi}^{r}, U\right)=(-1)^{m-d-1} I\left(\chi^{r}, x\right) \cdot E u(Z, x)=(-1)^{m-1} I\left(\chi^{r}, x\right) \cdot E \check{u}(Z, x)$, d'où le lemme.

4.1.7. Démonstration du théorème 4.1.5. On remarque que $E u_{f}(Z, x)$ ne dépend que de $j_{f(x)}^{*}(f \mid z)=\sum n_{i} A_{i}$, où $A_{i}$ sont les composantes irréductibles.

Le relèvement $\widehat{\chi}^{r}$ induit, par restriction sur chaque composante $C\left(A_{i}, M\right)$ de l'espace $C\left(\left.f\right|_{z}, M\right)_{f(x)}$, une $r$-section $\widehat{\chi}_{i}^{r}$ du fibré $E_{i}$, restriction du fibré $E$ au dessus de $U_{i}^{\prime}=U^{\prime} \cap C\left(A_{i}, M\right)$. D'après le lemme 4.1.6, on a

$$
\begin{gathered}
\operatorname{Obs}\left(\widehat{\chi}_{i}^{r}, U_{i}^{\prime}\right)=(-1)^{m-1} I\left(\chi^{r}, x\right) \cdot E \check{u}\left(A_{i}, x\right) . \\
\operatorname{Obs}\left(\widehat{\chi}^{r}, U^{\prime}\right)=\sum n_{i} \operatorname{Obs}\left(\widehat{\chi}_{i}^{r}, U_{i}^{\prime}\right)=(-1)^{m-1} I\left(\chi^{r}, x\right) \\
\cdot \sum n_{i} E \check{u}\left(A_{i}, x\right)=(-1)^{m-1} I\left(\chi^{r}, x\right) \cdot E \check{u}_{f}(Z, x) .
\end{gathered}
$$

D'où le théorème.

\subsection{Classe de Chern bivariante d'un fibré}

Soit $p$ un entier compris entre 0 et $d$. Soit $F$ un fibré vectoriel complexe de rang $n$ sur $X$ et $c^{p}(F)$ la classe de Chern usuelle.

4.2.1. Définition. La classe $c^{p}(F) \cdot[X]_{f}$ de $H^{2(-d+p)}(X \longrightarrow S)$ obtenue par produit bivariant est appelée classe de Chern bivariante de $F$.

Dans ce qui suit nous explicitons deux cocycles dans $H^{2(m-d+p)}(S \times$ $M, S \times M \backslash X)$ qui représentent la classe de Chern bivariante de $F$. On reprend les hypothèses et notations de $\S 2.6 .1$.

4.2.2. A partir d'un cocycle de la classe de Chern usuelle.

Un cocycle $\xi$ de la classe de Chern $c^{p}(F)$ dans $H^{2 p}(X)$ peut être obtenu comme suit : soit $r=n-p+1$, on peut construire $r$ sections de $F$ linéairement indépendantes $\chi^{r}$ sur le $2 p$-squelette de $\Delta(K)$ avec des singularités isolées à l'intérieur des $\Delta(K)$-cellules de dimension $2 p$. Notons $\lambda_{\varrho}$ l'indice de $\chi^{r}$ évalué sur la $2 p$-cellule $\varrho$, et $\delta(\varrho)$ la cochaîne élémentaire associée à $\varrho$, la classe de Chern $c^{p}(F)$ est représentable par le cocycle $\xi=\sum \lambda_{\varrho} \delta(\varrho)$, où $\varrho$ décrit les cellules de $\triangle(K)$ de dimension $2 p$. Les détails de construction et de raisonnement sont classiques [St]. Par abus de langage, on appelle $\chi^{r}$ un champ de $r$-repères. 
D'autre part, la classe fondamentale bivariante $[X]_{f}$ est une classe dans l'homologie bivariante

$$
H^{-2 d}(X \longrightarrow S)=H^{2(m-d)}(S \times M, S \times M \backslash X) .
$$

Le théorème 2.8.3 montre qu'un cocycle de cette classe cohomologique est $\Gamma=\sum \delta(D(\sigma))$ correspondant aux cellules $D(\sigma)$ de dimension $2(m-d+1)$, associées aux cellules $\sigma$ de dimension maximale dans $X$.

Dans ce cas particulier, le produit bivariant est défini par le cup produit

$$
H^{i}(X) \times H^{j}(S \times M,(S \times M) \backslash X) \longrightarrow H^{i+j}(S \times M,(S \times M) \backslash X) .
$$

Notons $\gamma=\xi$. $\Gamma$ le cup produit des deux cocycles. Supposons que l'orientation sur $\triangle(K)$ soit comme dans [Br2]. Alors le cocycle $\Gamma$ n'est autre que le cocycle $u^{2(m-d)}$ représentant la classe de Thom $([\mathrm{Br} 2])$ et le cup produit ci-dessus est l'homomorphisme de Thom.

Soit $\sigma$ une cellule de $K$ de dimension $2(d-p+1)$, la cellule duale $D(\sigma)$ est de dimension $2(m-d+p)$, et $D(\sigma) \cap X$ est de dimension $2 p$. Par définition du cup produit simplicial, l'évaluation $\langle\gamma, D(\sigma)\rangle$ du cocycle $\gamma$ sur $D(\sigma)$ est la somme des indices $\mu_{D(\sigma)}=\sum \lambda_{\varrho}$ de $\chi^{r}$ évalués sur les $2 p$-cellules $\varrho$ situées dans $D(\sigma) \cap X$. Le cocycle $\gamma$ est donc la somme $\sum \mu_{D(\sigma)} \delta(D(\sigma))$, où $\sigma$ décrit les cellules de $X$ de dimension relative $2(d-p+1)$, et $\delta(D(\sigma))$ désigne la $D(K)$-cochaîne élémentaire associée à $D(\sigma)$. On a donc le lemme suivant

LEMME. - La classe de Chern bivariante $c^{p}(F) \cdot[X]_{f}$ est représentable, dans $H^{2(m-d+p)}(S \times M,(S \times M) \backslash X)$, par le cocycle $\gamma=\sum \mu_{D(\sigma)} \delta(D(\sigma))$.

4.2.3. A partir d'un champ de repères relatifs.

Notons $\tilde{\chi}^{r}$ un champ relatif de $r$-repères défini sur les cellules duales relatives $A_{f}^{\prime}(\sigma)$ des cellules $\sigma$ de $K$ de dimension relative $2(d-p)$, sans singularité sur les bords relatifs $B_{f}^{\prime}(\sigma)$, et avec singularités à l'intérieur des $A_{f}^{\prime}(\sigma)$, isolées dans chaque fibre $A_{f}^{\prime}(\sigma)_{y}$. Notons $\mu_{\sigma}$ l'indice du champ $\tilde{\chi}^{r}$ sur $A_{f}^{\prime}(\sigma)_{y}$, il ne dépend que de la cellule $\sigma$. On obtient ainsi un cocycle bivariant $\gamma^{\prime}=\sum \mu_{\sigma} \delta_{b i v}\left(A_{f}(\sigma)\right)$ (voir $\left.\S 2.6\right)$, où $\delta_{b i v}\left(A_{f}(\sigma)\right.$ ) est la cochaîne élémentaire dans $C^{2(m-d+p)}(S \times M, S \times M \backslash X)$ prenant la valeur 1 sur chaque fibre $A_{f}(\sigma)_{y}$.

Considérons une cellule $\sigma$ de $K$ de dimension $2(d-p+1)$, il y a deux cas possibles : 
1. Ou bien la dimension relative de $\sigma$ est $2(d-p)$, c'est-à-dire $\tau=f(\sigma)$ est de dimension maximale 2 dans $L$ ( $S$ est de dimension réel 2). D'après l'assertion 3) du lemme 2.3.5, on a $D(\sigma)=A_{f}(\sigma)_{\hat{\tau}}=D\left(\sigma_{\hat{\tau}}\right)$.

2. Ou bien la dimension relative de $\sigma$ est plus grande que $2(d-p)$, alors il existe une cellule $\sigma^{\prime}$ de dimension relative $2(d-p)$, face de $\sigma$, telle que $f(\sigma)=f\left(\sigma^{\prime}\right)=\tau$. D'après le lemme 2.3.3, on a,

$$
D(\sigma) \subset A_{f}(\sigma) \subset B_{f}\left(\sigma^{\prime}\right) .
$$

La restriction du champ relatif $\tilde{\chi}^{r}$ aux cellules $D(\sigma)$ est un champ "absolu" $\chi^{r}$ qui sert à obtenir un cocycle de la classe de Chern $c^{p}(F)$.

Dans le cas $1, \chi^{r}$ est à singularités isolées en point $\hat{\sigma}$ de $D(\sigma)$. L'indice $I\left(\chi^{r}, \hat{\sigma}\right)$ est égal à l'indice relatif $\mu_{\sigma}$ du champ $\tilde{\chi}^{r}$.

Dans le cas 2, $\chi^{r}$ est sans singularité sur $D(\sigma)$. L'indice $I\left(\chi^{r}, D(\sigma)\right)$ est donc nul.

Un champ relatif $\tilde{\chi}^{r}$ de $r$-repères détermine, d'après Lemme 4.2.2, un cocycle $\gamma$ de la classe de Chern bivariante $c^{p}(F) \cdot[X]_{f}$ dans $H^{2(m-d+p)}(S \times$ $M, S \times M \backslash X)$.

On peut évaluer le cocycle $\gamma$ sur une cellule duale relative $A_{f}(\sigma)$ de manière "bivariante" :

$<\gamma, A_{f}(\sigma)>_{b i v}:=<\gamma, A_{f}(\sigma)_{y}>$, où $y$ est un point général de $f(\sigma)$. Cette évaluation ne dépend pas de la fibre choisie, elle est égale à l'indice $\mu_{\sigma}$. Ce cocycle $\gamma$ est donc le même cocycle $\gamma^{\prime}=\sum \mu_{\sigma} \delta_{b i v}\left(A_{f}(\sigma)\right)$.

LEMME. - La classe de Chern bivariante $c^{p}(F) \cdot[X]_{f}$ est représentable par le cocycle $\gamma^{\prime}=\sum \mu_{\sigma} \delta_{b i v}\left(A_{f}(\sigma)\right)$, où $\sigma$ décrit les cellules de $X$ de dimension relative $2(d-p)$.

\subsection{Démonstration du théorème principal}

Il suffit de montrer le résultat pour le cas où le cycle $Z$ est irréductible. Soient $d$ la dimension relative $Z$ et $p$ un entier compris entre 0 et $d$. Notons $g$ le morphisme composé $f \circ \nu_{f}: N_{f}(Z) \longrightarrow S$.

D'après le lemme 2.2 .5 et les hypothèses du théorème, il existe des décompositions cellulaires $K$ et $\widetilde{K}$ de $S \times M$ et $S \times G_{d}(T M)$ compatibles avec $Z$ et $N_{f}(Z)$ respectivement, et on peut construire les cellules duales relatives. Prenons les notations de $§ 2.4$. 
D'après le lemme 2.6.2, la classe de Chern bivariante de Brasselet $c_{B}^{p}(\alpha)$ est représentable dans $H^{2(m-r+1)}(S \times M, S \times M \backslash X)$ par le cocycle :

$$
\gamma(\alpha)=\sum_{\sigma} \alpha(\sigma) \cdot \mu_{\sigma} \cdot \delta_{b i v}\left(A_{f}(\sigma)\right)
$$

et ce dernier est obtenu à partir d'un champ radial relatif $\chi^{r}$.

D'autre part, la classe de Chern bivariante de Sabbah correspondant au cycle $Z$ est donnée par

$$
c_{S}^{p}(Z)=I n c_{*} \nu_{f *}\left(c^{p}(\theta) \cdot\left[N_{f}(Z)\right]_{g}\right) \in H^{-2 p}(X \longrightarrow S) .
$$

On va montrer que le cocycle $\gamma(\alpha)$ est un représentant de la classe $c_{S}^{p}(Z)$.

D'après la proposition 2.7.1, on peut relever le champ $\chi^{r}$ en $r$ sections linéairement indépendantes $\tilde{\chi}^{r}$ du fibré $\theta$, sans singularité sur les $\nu_{f}^{-1}\left(B_{f}^{\prime}(\sigma)\right)$. D'après la proposition 2.4 .3 , on peut étendre le relèvement $\widetilde{\chi}^{r}$ à l'intérieur des cellules duales relatives $A_{g}(\tilde{\sigma})$ avec des singularités sur les $C_{g}(\tilde{\sigma})$. On note encore $\tilde{\chi}^{r}$ l'extension de ce relèvement. C'est un champ à singularités isolées dans chaque fibre du morphisme $g$.

D'après le lemme 4.2.3, le relèvement étendu $\widetilde{\chi}^{r}$ du champ $\chi^{r}$ détermine un cocycle $\tilde{\gamma}$ de la classe $c^{p}(\theta) \cdot\left[N_{f}(Z)\right]_{g}$. Ce cocycle s'écrit

$$
\tilde{\gamma}=\sum_{\tilde{\sigma}} \mu_{\tilde{\sigma}} \delta_{b i v}\left(A_{g}(\tilde{\sigma})\right)
$$

où la somme porte sur toutes les cellules $\tilde{\sigma}$ de dimension relative $2(r-1)$ de la décomposition cellulaire $\widetilde{K}$ de $S \times G_{d}(T M)$.

D'après la proposition 2.4.3, pour chaque cellule $\sigma$ de dimension relative $2(r-1)$, on a $\nu_{f}^{-1}\left(A_{f}^{\prime}(\sigma)\right)=\bigcup_{\tilde{\sigma} \in h(\sigma)} A_{g}^{\prime}(\tilde{\sigma})$, où $h(\sigma)$ est l'ensemble des cellules $\nu_{f}$-horizontales sur $\sigma$. On a donc

$$
\begin{aligned}
\nu_{f *}(\tilde{\gamma}) & =\nu_{f *}\left(\sum_{\tilde{\sigma}} \mu_{\tilde{\sigma}} \delta_{b i v}\left(A_{g}(\tilde{\sigma})\right)\right)=\nu_{f *}\left(\sum_{\sigma} \sum_{\tilde{\sigma} \in h(\sigma)} \mu_{\tilde{\sigma}} \delta_{b i v}\left(A_{g}(\tilde{\sigma})\right)\right) \\
& =\sum_{\sigma}\left(\sum_{\tilde{\sigma} \in h(\sigma)} \mu_{\tilde{\sigma}}\right) \delta_{b i v}\left(A_{f}(\sigma)\right)
\end{aligned}
$$

où, dans les doubles sommes, la première porte sur toutes les cellules $\sigma$ de $K$ de dimension relative $2(r-1)$. La deuxième égalité provient du fait que 
les singularités de $\tilde{\chi}^{r}$ sont à l'intérieur des $A_{g}(\tilde{\sigma})$ et se montre comme dans [BS, Th. 6.1].

Le cocycle $\gamma^{\prime}=\nu_{f *}(\tilde{\gamma})$ est un cocycle de la classe $c_{S}^{p}(Z)$. La somme des indices $\sum_{\tilde{\sigma} \in h(\sigma)} \mu_{\tilde{\sigma}}$ est l'obstruction à étendre la restriction $\tilde{\chi}_{y}^{r}$ du champ $\tilde{\chi}^{r}$ sur une fibre au dessus d'un point $y$, à l'intérieur de $\nu_{f}^{-1}\left(A_{f}^{\prime}(\sigma)_{y}\right) \cap N_{f}(Z)$, pour $y$ à l'intérieur de $f(\sigma)$. Le théorème 4.1.5 de proportionnalité montre que cette obstruction est égale au produit $I\left(\chi^{r}, x\right) \cdot E u_{f}(Z, x)$. On a donc

$$
\sum_{\tilde{\sigma} \in h(\sigma)} \mu_{\tilde{\sigma}}=I\left(\chi^{r}, x\right) \cdot E u_{f}(Z, x)=\mu_{\sigma} \cdot E u_{f}(Z, x)=\mu_{\sigma} \cdot \alpha(\sigma),
$$

puisque le nombre $\mu_{\sigma}$ est par définition, l'indice $I\left(\chi^{r}, x\right)$ du champ $\chi^{r}$ en un point $x$ de $C_{f}(\sigma)$. Ceci montre que l'évaluation "bivariante" du cocycle $\gamma^{\prime}$ sur $A_{f}(\sigma)$, c'est-à-dire $\left\langle\gamma^{\prime}, A_{f}(\sigma)_{y}>\right.$ pour $y$ dans l'intérieur de $f(\sigma)$, est égale à $\mu_{\sigma} \cdot \alpha(\sigma)$. On en déduit donc que le cocycle $\gamma^{\prime}$ coïncide avec le cocycle $\gamma(\alpha)$, ce qui termine la démonstration.

\section{Bibliographie}

[Ba1] BARLeT (D.). - Espace analytique réduit des cycles analytiques complexes compacts d'un espace analytique complexe de dimension finie. Séminaire Norguet. Lecture Notes 482, p. 1-158.

[Ba2] BARLET (D.). - Familles analytiques de cycles et classes fondamentales relatives. Lecture Notes 807, p.1-24.

[Br1] Brasselet (J.-P.). - Existence des classes de Chern en théorie bivariante. Astérisque 101-102 (1983).

[Br2] Brasselet (J.-P.). - Définition combinatoire des homomorphismes de Poincaré, Alexander et Thom, pour une pseudo-variété. Astérisque 82-83 (1981).

[BS] Brasselet (J.-P.) et SchwarTZ (M.H.). - Sur les classes de Chern d'un ensemble analytique complexe. Astérisque 82-83 (1981).

[EOY] ERNSTRÖM (L.), OHMOTo (T.) et YokURA (S.). - On topological Radon transformations. J. of Pure and Applied Algebra, 120 (1997), p. 235 - 254.

[FM] Fulton (W.), MACPherson (R.). - Categorical frame work for the study of singular spaces. Mem. Amer. Math. Soc., 243 (1981).

[FP] Fritsch (R.), PiCCININI (R.A.). - Cellular structures in topology. Cambridge Univ. Press (1990).

[GS] Gonzalez-SPRINBerg (G.). - L'obstruction locale d'Euler et le théorème de MacPherson. Astérisque 82-83 (1981).

[Hi1] HironaKa (H.). - Flattening theorem in complex analytic geometry. Amer. J. of Math. Vol. 97, N $^{\circ}$. 2, (1975).

[Hi2] Hironaka (H.). - Stratifications and Flatness. in Real and Complex Singularities (Nordic Summer School, Oslo, 1976; Sijthoff and Noordhoff, 1977). 
[HMS] Henry (J.-P.), Merle (M.), SABbah (C.). - Sur la condition de Thom stricte pour un espace analytique complexe. Annales de l'E.N.S.(1984).

[Ka] KAdDAR (M.). - Classe fondamentale relative en cohomologie de DeligneBeilinson. Thèse, préprint, Université de Nancy, (1994).

[LT] LÊ (D.T.) et TEISSIER (B.). - Variétés polaires locales et classes de Chern des variétés singulières. Annals of Math. 114 (1981), p. $457-491$.

[Mun] Munkres (J.R.). - Elements of algebraic topology. Addison-Wesley Publishing Company (1984).

[Sab1] SABBAH (C.).- Morphismes analytiques stratifiés sans éclatement et cycles évanescents. Astérisque 101-102.

[Sab2] SABBAH (C.). - Quelques remarques sur la géométrie des espaces conormaux. Astérisque 130.

[Sab3] SABbah (C.). - Espaces conormaux bivariants. Thèse, Ecole polytechnique, Paris, (1986).

[Sab4] SABbaH (C.). - Quelques remarques sur la théorie des classes de Chern des espaces analytiques singuliers. Ecole polytechnique, Paris, (1986).

[St] Steenrod (N.). - The topology of fibre bundles. Princeton Univ. Press (1951).

[Te1] TEISSIER (B.). - Sur la triangulation des morphismes sous-analytiques. Rapport de recherche, E.N.S. (1986).

[Te2] TeIssier (B.). - Variétés polaires II. Actes de la Conférence de La Rabida, Lecture Notes 961, Springer-Verlag, p. $314-491$.

[Ve1] VERDIER (J.-L.). - Spécialisation des classes de Chern. Astérisque 82-83.

[Ve2] Verdier (J.-L.). - Séminaire de Géométrie analytique, Astérisque 36-37, exp.VI.

[Ve3] VERdier (J.-L.). - Dualité dans la cohomologie des espaces localement compacts. Séminaire Bourbaki 300 (1965).

[Yo1] YoKURA (S.). - Homological Verdier-Radon functors on smooth varieties. Sci. Rep. Kagoshima Univ., 45 (1996), p. 89 - 104.

[Yo2] YoKuRA (S.). - On a Verdier-type Riemann-Roch for Chern-SchwartzMacPherson class. Topology and its Applications, 20 (1998), p. $1-13$.

[Zh] Zhou (J.). - Classes de Chern en Théorie bivariante. Thèse, Université d'AixMarseille II, (1995). 